J. DIFFERENTIAL GEOMETRY

76 (2007) 317-349

\title{
THE CALABI-YAU EQUATION ON ALMOST-KÄHLER FOUR-MANIFOLDS
}

\author{
BEN WEINKOVE
}

\begin{abstract}
Let $(M, \omega)$ be a compact symplectic 4-manifold with a compatible almost complex structure $J$. The problem of finding a $J$-compatible symplectic form with prescribed volume form is an almost-Kähler analogue of Yau's theorem and is connected to a programme in symplectic topology proposed by Donaldson. We call the corresponding equation for the symplectic form the CalabiYau equation. Solutions are unique in their cohomology class. It is shown in this paper that a solution to this equation exists if the Nijenhuis tensor is small in a certain sense. Without this assumption, it is shown that the problem of existence can be reduced to obtaining a $C^{0}$ bound on a scalar potential function.
\end{abstract}

\section{Introduction}

In 1954 Calabi $[\mathbf{C a}$ ] conjectured that any representative of the first Chern class of a compact Kähler manifold $(M, \omega)$ can be written as the Ricci curvature of a Kähler metric $\omega^{\prime}$ cohomologous to $\omega$. He showed that any such metrics are unique. Yau [Ya] famously solved Calabi's conjecture around twenty years later. This result, and the immediate corollary that any Kähler manifold with $c_{1}(M)=0$ admits a Ricci-flat metric, have had many applications in both mathematics and theoretical physics.

Yau's theorem is equivalent to finding a Kähler metric in a given Kähler class with prescribed volume form. By the $\partial \bar{\partial}$-Lemma this amounts to solving the complex Monge-Ampère equation

$$
(\omega+\sqrt{-1} \partial \bar{\partial} \phi)^{n}=e^{F} \omega^{n},
$$

for smooth real $\phi$ with $\omega+\sqrt{-1} \partial \bar{\partial} \phi>0$, where $n=\operatorname{dim}_{\mathbb{C}} M$ and $F$ is any smooth function with $\int_{M} e^{F} \omega^{n}=\int_{M} \omega^{n}$. Yau solved this equation by considering the family of equations obtained by replacing $F$ by $t F+c_{t}$ for some constant $c_{t}$, for $t \in[0,1]$ and using the continuity method. This

The author is supported in part by National Science Foundation grant DMS-0504285. This work was carried out while the author was visiting Imperial College, London on a Royal Society Research Assistantship.

Received 05/09/2006. 
requires an openness argument using the implicit function theorem, and, more importantly, a closedness argument which requires his celebrated a priori estimates. Yau also generalized Calabi's conjecture: first in the case when the right hand side of (1.1) may have poles or zeros [Ya]; and second, with Tian, in the context of complete non-compact Kähler Ricci-flat metrics [TiYa1, TiYa2]. For other results along these lines, see $[\mathbf{K o}]$, [BaKo1], [BaKo2], [Jo], for example.

The aim of this paper is to attempt to generalize Yau's theorem in a very different direction. We consider the case when the almost complex structure is not integrable. This problem was suggested to the author by Donaldson and is motivated by a wider programme of his on the symplectic topology of 4 -manifolds [Do]. Let $(M, \omega)$ be a symplectic four-manifold. Then there exists an almost complex structure $J$ which is compatible with $\omega$. This defines a metric $g$ by

$$
g(\cdot, \cdot)=\omega(\cdot, J \cdot)>0 .
$$

If $J$ is integrable then it is Kähler. In general, the data $(M, \omega, J)$ is called an almost-Kähler manifold and we will call $\omega$ an almost-Kähler form. The volume form version of Yau's theorem still makes sense. Given an almost-Kähler 4-manifold $(M, \omega, J)$ we ask whether there exists an almost-Kähler form $\omega^{\prime}$ solving the equation

$$
\omega^{\prime 2}=e^{F} \omega^{2},
$$

for any function $F$ satisfying

$$
\int_{M} e^{F} \omega^{2}=\int_{M} \omega^{2},
$$

and we also ask whether $\omega^{\prime}$ can be taken to be cohomologous to $\omega$. We call (1.2) the Calabi-Yau equation. Any solution to (1.2) is unique in its cohomology class - this fact was pointed out to the author by Donaldson. A proof is given in section 2 .

Following Yau, we use the continuity method to try to obtain the existence of a solution. First, we consider the question of a priori estimates for solutions to (1.2). For simplicity, assume that $\omega^{\prime}$ is cohomologous to $\omega$. We show that all the estimates can be reduced to a uniform bound of a scalar potential function $\phi_{1}$ defined, up to a constant, by

$$
\frac{\omega \wedge \omega^{\prime}}{\omega^{\prime 2}}=1-\frac{1}{4} \Delta^{\prime} \phi_{1},
$$

where $\Delta^{\prime}$ is the Laplacian associated to $\omega^{\prime}$. The function $\phi_{1}$ belongs to a a 1-parameter family of 'almost-Kähler potentials' $\left\{\phi_{s}\right\}_{s \in[0,1]}$, defined in section 2, which all coincide in the Kähler case with the usual Kähler potential.

Theorem 1. Let $(M, \omega, J)$ be a compact almost-Kähler 4-manifold. Suppose that $\omega^{\prime}$ is another almost-Kähler form, cohomologous to $\omega$, and 
satisfying (1.2). Then there exist positive constants $K_{i}$ depending only on $(M, \omega, J), F$ and $\operatorname{osc}_{M} \phi_{1}$ such that $\omega^{\prime} \geq K_{0}^{-1} \omega$ and

$$
\left\|\omega^{\prime}\right\|_{C^{i}(g)} \leq K_{i} \quad \text { for } i=0,1,2, \ldots,
$$

where $\operatorname{osc}_{M} \phi_{1}=\sup _{M} \phi_{1}-\inf _{M} \phi_{1}$.

An analogous result holds even if $\omega^{\prime}$ and $\omega$ are not necessarily cohomologous (see Section 7). We turn now to the question of openness in the continuity method. Denote by $\mathcal{H}_{\omega}^{+}$the space of self-dual harmonic 2 -forms with respect to $\omega$ and by $H_{\omega}^{+}$the corresponding subspace in $H^{2}(M ; \mathbb{R}) . H_{\omega}^{+}$is a maximal positive subspace for the intersection form on $H^{2}(M ; \mathbb{R})$ and its dimension is $b^{+}(M)$. Notice that $\omega$ is harmonic and self-dual and so $b^{+}(M) \geq 1$. If $b^{+}(M)=1$ then we can show the openness part of the continuity method, remaining in the same cohomology class. In the case where $b^{+}(M)>1$ the openness argument still works if we allow the class to vary within $H_{\omega}^{+}$.

Under the assumption that the Nijenhuis tensor $N(J)$ is small in the $L^{1}$ norm, the required uniform bound on $\phi_{1}$ can be obtained. So in this case, we can solve equation (1.2).

Theorem 2. Let $(M, \omega, J)$ be a compact almost Kähler 4-manifold.

(i) Suppose $b^{+}(M)=1$. Then for $F \in C^{\infty}(M)$ satisfying (1.3) there exists an almost-Kähler form $\omega^{\prime}$ cohomologous to $\omega$ solving (1.2) if

$$
\|N(J)\|_{L^{1}(g)}<\epsilon
$$

for $\epsilon>0$ depending only on $g$ and $\|F\|_{C^{2}(g)}$.

(ii) If $b^{+}(M)>1$ then the same holds except that the solution $\omega^{\prime}$ may lie in a different cohomology class in $H_{\omega}^{+}$.

With a little work, an explicit $\epsilon$ could be written down. However, it is hoped that the condition (1.5) could be removed entirely (cf. [Do]). In addition, it would be interesting to improve on Theorem 2 even further in light of a possible application in symplectic topology described to the author by Donaldson. Given an almost complex structure $J_{1}$ on a symplectic 4-manifold, a natural question is: does there exist a symplectic form compatible with $J_{1}$ ? In general the answer is negative, as can be seen from the well-known Kodaira-Thurston example [Th], [FeGoGr]. However, it is sensible to ask this question under the (obviously necessary) assumption that there exists some symplectic form $\Omega$ taming $J_{1}$. In this case, there exists an almost complex structure $J_{0}$ compatible with $\Omega$ and, by a well-known result of Gromov $[\mathbf{G r}]$, a smooth path of almost complex structures $\left\{J_{t}\right\}_{t \in[0,1]}$ all taming $\Omega$. Set $\omega_{0}=\Omega$ and consider the equation

$$
\omega_{t}^{2}=\Omega^{2}
$$


for $\omega_{t}$ compatible with $J_{t}$. Finding a solution for $t=1$ would solve the problem. To prove this using a continuity method one would require estimates for $\omega_{t}$ depending only on $\Omega$ and $J_{t}$.

These methods appear to make sense only in four dimensions, since the system of equations is overdetermined in higher dimensions. Nevertheless, it should be noted that many of the estimates here carry over easily to any dimension.

The outline of the paper is as follows: in Section 2, some preliminaries are given, almost-Kähler potentials are defined and uniqueness for the Calabi-Yau equation is proved; in Section 3 an estimate on the metric $g^{\prime}$ in terms of the potential is given; a Hölder estimate on the metric is proved in Section 4; the higher order estimates and the proof of Theorem 1 are given in Section 5; finally, in Sections 6 and 7, Theorem 2 is proved in the cases $b^{+}(M)=1$ and $b^{+}(M)>1$ respectively.

Remark 1.1. Delanoë [De] considered, following a suggestion of Gromov, a different problem concerning the equation (1.2). He looked for solutions of $\omega^{\prime n}=e^{F} \omega^{n}$, on an almost-Kähler manifold $(M, \omega)$ of dimension $2 n$, of the form $\omega^{\prime}=\omega+d(J d \phi)$ for a smooth real function $\phi$ so that $\omega^{\prime}$ tames $J$ but is not necessarily compatible with $J$ (here, $J$ acts on 1-forms in the usual way). He showed that when $n=2$, if there exists such a solution for every $F$, then $J$ is in fact integrable. We do not expect the solutions we obtain in Theorem 2 to be, in general, of the form $\omega^{\prime}=\omega+d(J d \phi)$ for any $\phi$.

Acknowledgements. The author is very grateful to: Simon Donaldson for suggesting this problem and for many subsequent helpful, insightful and encouraging discussions; S.-T. Yau whose original paper [Ya] made this work possible and whose lectures and discussions at Harvard University have been an invaluable source of inspiration; the author's former advisor D.H. Phong for his continued support and encouragement; Richard Thomas for some very useful conversations and for his help in arranging the author's year-long visit to Imperial College; Xiuxiong Chen, Joel Fine, Mark Haskins, Tom Mrowka, Jian Song and Valentino Tosatti for some helpful discussions.

\section{Almost-Kähler geometry and the Calabi-Yau equation}

\section{Notation and preliminaries.}

We will often work in local coordinates, making use of the Einstein summation convention. The almost complex structure $J=J_{i}{ }^{j} d x^{i} \otimes \frac{\partial}{\partial x^{j}}$ satisfies, by definition, the condition

$$
J_{i}^{k} J_{k}^{j}=-\delta_{i}^{j} .
$$

We will lower indices in the usual way using the metric $g$ so that

$$
J_{i j}=J_{i}{ }^{k} g_{k j}=\omega_{i j} \text {. }
$$


The condition $d \omega=0$ can be written as

$$
\partial_{i} J_{j k}+\partial_{j} J_{k i}+\partial_{k} J_{i j}=0 .
$$

It follows that the equation

$$
\nabla_{i} J_{j}{ }^{i}=0
$$

holds on an almost-Kähler manifold, where $\nabla$ is the Levi-Civita connection associated to the metric $g$. This implies that $\omega$ is harmonic with respect to the metric $g$.

Define two tensors $\mathcal{P}$ and $\mathcal{Q}$ by

$$
\begin{aligned}
\mathcal{P}_{k l}^{i j} & =\frac{1}{2}\left(\delta_{k}^{i} \delta_{l}^{j}-J_{k}{ }^{i} J_{l}{ }^{j}\right) \\
\mathcal{Q}_{k l}^{i j} & =\frac{1}{2}\left(\delta_{k}^{i} \delta_{l}^{j}+J_{k}{ }^{i} J_{l}{ }^{j}\right) .
\end{aligned}
$$

Then the compatibility of $g$ and $\omega$ with $J$ implies that $\mathcal{P}_{k l}^{i j} g_{i j}=0$ and $\mathcal{P}_{k l}^{i j} J_{i j}=0$. Considering $\mathcal{P}$ and $\mathcal{Q}$ as operators on two-tensors, we have

$$
\mathcal{P}+\mathcal{Q}=\mathrm{Id} .
$$

Moreover, at each point, $\mathcal{P}$ and $\mathcal{Q}$ are self-adjoint with respect to $g$ and define projections onto the spaces $\operatorname{ker} \mathcal{Q}$ and $\operatorname{ker} \mathcal{P}$ respectively.

The obstruction to the almost complex structure $J$ being integrable is the Nijenhuis tensor $N: T M \times T M \rightarrow T M$, which is given by

$$
N(X, Y)=[X, Y]+J[J X, Y]+J[X, J Y]-[J X, J Y] .
$$

In local coordinates, this can be written as

$$
N_{j k}^{i}=J_{k}{ }^{l} \partial_{l} J_{j}{ }^{i}+J_{l}{ }^{i} \partial_{j} J_{k}{ }^{l}-J_{j}{ }^{l} \partial_{l} J_{k}{ }^{i}-J_{l}{ }^{i} \partial_{k} J_{j}{ }^{l} .
$$

On an almost-Kähler manifold, the Nijenhuis tensor can be written in the simpler form

$$
N_{j k}^{i}=2\left(\nabla^{i} J_{j}^{l}\right) J_{k l} .
$$

By the Newlander-Nirenberg theorem, the almost complex structure $J$ is integrable if and only if $N$ vanishes identically and if and only if $\nabla J$ vanishes identically.

For later use, we also make the following simple observation. Let Rm denote the Riemmanian curvature tensor of an almost-Kähler metric $g$. Then

$$
\sup _{M}|\nabla J|^{2} \leq C\|\operatorname{Rm}\|_{C^{0}(g)}
$$

for $C$ a constant depending only on dimension. Indeed, using the usual commutation formulae for covariant derivatives along with (2.1) and $(2.2)$,

$$
0=\Delta|J|^{2}=2|\nabla J|^{2}+\mathrm{Rm} * J * J,
$$

where $*$ denotes some bilinear operation involving tensor products and the metric $g$. Similarly, by calculating $\Delta|\nabla J|^{2}$ we see that

$|\nabla \nabla J|^{2}=\nabla \nabla \mathrm{Rm} * J * J+\mathrm{Rm} * \nabla \nabla J * J+\nabla \mathrm{Rm} * J * \nabla J+\mathrm{Rm} * \nabla J * \nabla J$, 
and it follows that $\sup _{M}|\nabla \nabla J|^{2}$ can be bounded by a constant depending only on dimension and $\|\mathrm{Rm}\|_{C^{2}(g)}$.

\section{Almost-Kähler potentials.}

Now restrict to four dimensions. Let $\omega$ and $\omega^{\prime}$ be two almost-Kähler forms with $[\omega]=\left[\omega^{\prime}\right]$. For $s \in[0,1]$, let $\Omega_{s}=(1-s) \omega+s \omega^{\prime}$ and define the almost-Kähler potentials $\phi_{s}$ by

$$
(1-2 s) \omega \wedge \omega^{\prime}+s \omega^{\prime 2}-(1-s) \omega^{2}=-\frac{1}{2} \Omega_{s} \wedge d\left(J d \phi_{s}\right) .
$$

Since $-\Omega \wedge d(J d \phi)=\frac{1}{2} \Delta_{\Omega} \phi \Omega^{2}$ for any almost-Kähler form $\Omega$ and function $\phi$, the existence of the $\phi_{s}$ follows from elementary Hodge theory. The $\phi_{s}$ are uniquely determined up to the addition of a constant. In the Kähler case, they all coincide with the usual Kähler potential $\phi$ given by $\omega^{\prime}=\omega+\sqrt{-1} \partial \bar{\partial} \phi$. We are interested in three particular almost-Kähler potentials, corresponding to $s=0, \frac{1}{2}, 1$. They satisfy:

$$
\begin{aligned}
\frac{1}{4} \Delta \phi_{0} & =\frac{\omega \wedge \omega^{\prime}}{\omega^{2}}-1 \\
\frac{1}{4} \Delta^{\prime} \phi_{1} & =1-\frac{\omega \wedge \omega^{\prime}}{\omega^{\prime 2}} \\
\frac{1}{2}\left(\Delta_{\frac{1}{2}} \phi_{\frac{1}{2}}\right) & =\frac{\omega^{\prime 2}-\omega^{2}}{\Omega_{\frac{1}{2}}^{2}},
\end{aligned}
$$

where $\Delta^{\prime}$ and $\Delta_{\frac{1}{2}}$ are the Laplacians associated to $\omega^{\prime}$ and $\Omega_{\frac{1}{2}}$.

In addition, for each $s$, define a one form $a_{s}$ by the equations

$$
\omega^{\prime}=\omega-\frac{1}{2} d\left(J d \phi_{s}\right)+d a_{s},
$$

and $d_{s}^{*} a_{s}=0$, where $d_{s}^{*}$ is the formal adjoint of $d$ associated to the metric $\Omega_{s}$. Note that $a_{s}$ is defined only up to the addition of a harmonic 1-form. A short calculation shows that $a_{s}$ satisfies the elliptic system

$$
\left\{\begin{aligned}
d a_{s} \wedge \Omega_{s} & =0 \\
\mathcal{P} d a_{s} & =\frac{1}{4}\left(\partial_{i} J_{j}{ }^{k}-\partial_{j} J_{i}{ }^{k}\right)\left(\partial_{k} \phi_{s}\right) d x^{i} \wedge d x^{j} \\
d_{s}^{*} a_{s} & =0 .
\end{aligned}\right.
$$

It will be convenient to give a different formulation of (2.6). Let $*_{s}$ be the Hodge-star operator associated to $\Omega_{s}$. Then the projection $\frac{1}{2}\left(1+*_{s}\right)$ : $\Lambda^{2} \rightarrow \Lambda_{s}^{+}$onto the self-dual two forms can be written

$$
\frac{1}{2}\left(1+*_{s}\right)(\chi)=\left(\frac{\Omega_{s} \wedge \chi}{\Omega_{s}^{2}}\right) \Omega_{s}+\mathcal{P} \chi, \quad \text { for } \chi \in \Lambda^{2} .
$$

Hence, the system (2.6) can be rewritten as

$$
\left\{\begin{aligned}
d_{s}^{+} a_{s} & =\frac{1}{4}\left(\partial_{i} J_{j}{ }^{k}-\partial_{j} J_{i}{ }^{k}\right)\left(\partial_{k} \phi_{s}\right) d x^{i} \wedge d x^{j} \\
d_{s}^{*} a_{s} & =0
\end{aligned}\right.
$$


for $d_{s}^{+}=\frac{1}{2}\left(1+*_{s}\right) d: \Lambda^{1} \rightarrow \Lambda_{s}^{+}$. Observe that, since $d_{s}^{+} b=0$ implies $d b=0$ for any 1 -form $b$ (see for example [DoKr], Prop. 1.1.19), the kernel of the operator $\left(d_{s}^{+}, d_{s}^{*}\right)$ consists of the harmonic 1-forms.

\section{The Calabi-Yau equation.}

For a manifold of dimension $n=2 m$, linearizing the Calabi-Yau equation gives $a \mapsto \omega^{m-1} \wedge d a$ for a one-form $a$. Combining with the linear operator $a \mapsto \mathcal{P} d a$ and imposing $d^{*} a=0$ gives a system which is elliptic if $n=4$ and overdetermined if $n>4$.

Restricting to four-manifolds, solutions to (1.2) are unique in their cohomology class. To see this, let $\omega_{1}$ and $\omega_{2}=\omega_{1}+d b$ be cohomologous almost-Kähler forms with $\omega_{1}^{2}=\omega_{2}^{2}$. Let $\Omega=\omega_{1}+\omega_{2}$. Then $\Omega \wedge d b=0$ and $\mathcal{P} d b=0$ from which it follows that $d_{\Omega}^{+} b=0$. Then $d b=0$ and so $\omega_{1}=\omega_{2}$.

Finally we mention that the Calabi-Yau equation (1.2) can also be written as

$$
\operatorname{tr}_{g} g^{\prime}=e^{F} \operatorname{tr}_{g^{\prime}} g,
$$

where we are writing $g^{\prime}$ for the metric associated to $\omega^{\prime}$ and where

$$
\operatorname{tr}_{g} g^{\prime}=g^{i j} g_{i j}^{\prime}=J^{i j} J_{i j}^{\prime}, \quad \text { and } \operatorname{tr}_{g^{\prime}} g=g^{i j} g_{i j}=J^{\prime i j} J_{i j} .
$$

We are using here the obvious notation for lowering and raising indices using the metric $g^{\prime}$, so that $J_{i j}^{\prime}=J_{i}{ }^{k} g_{k j}^{\prime}$ and $J^{\prime i j}=J_{k}{ }^{\prime} g^{\prime k i}$.

\section{Uniform estimate on the metric}

In this section we will prove an estimate on the metric in terms of $\phi_{1}$, similar to the one proved by Yau [Ya] (see also [Au]) in the Kähler case. The computations here are somewhat more involved because of extra terms arising from the non-integrability of the almost complex structure.

Theorem 3.1. There exist constants $C$ and $A$ depending only on $\|\operatorname{Rm}(g)\|_{C^{2}(g)}, \sup _{M}|F|$ and the lower bound of $\Delta F$ such that

$$
\operatorname{tr}_{g} g^{\prime} \leq C e^{A\left(\phi_{1}-\inf _{M} \phi_{1}\right)} .
$$

Proof. We will calculate $\Delta^{\prime}\left(\log \left(\operatorname{tr}_{g} g^{\prime}\right)-A \phi_{1}\right)$, where $A$ is a constant to be determined, and then apply the maximum principle. For this calculation we will work at a point using normal coordinates for $g$.

First, the equation (1.2) can be written

$$
\log \operatorname{det} g^{\prime}=2 F+\log \operatorname{det} g .
$$

Applying the Laplace operator $\Delta$ of the metric $g$, we obtain

$$
2 \Delta F=g^{i j} g^{\prime k l} \partial_{i} \partial_{j} g_{k l}^{\prime}-g^{i j} g^{\prime k q} g^{\prime p l}\left(\partial_{i} g_{p q}^{\prime}\right)\left(\partial_{j} g_{k l}^{\prime}\right)-g^{i j} g^{k l} \partial_{i} \partial_{j} g_{k l} \text {. }
$$


Now calculate

$$
\Delta^{\prime}\left(\operatorname{tr}_{g} g^{\prime}\right)=g^{\prime k l} g^{i j} \partial_{k} \partial_{l} g_{i j}^{\prime}+g^{\prime k l} g_{i j}^{\prime} \partial_{k} \partial_{l} g^{i j}-g^{\prime k l} \Gamma_{k l}^{\prime p} g^{i j} \partial_{p} g_{i j}^{\prime},
$$

where we use $\Gamma_{k l}^{\prime p}$ to denote the Christoffel symbols corresponding to $\nabla^{\prime}$. We can rewrite this last term using the equation (valid at a point)

$$
g^{\prime k l} \Gamma_{k l}^{\prime p}=-J_{q}{ }^{p} g^{\prime k l} \nabla_{k} J_{l}^{q},
$$

which follows from the equation $g^{\prime k l} \nabla_{k}^{\prime} J_{l}^{q}=0$. Thus we have

$$
\Delta^{\prime}\left(\operatorname{tr}_{g} g^{\prime}\right)=g^{\prime k l} g^{i j} \partial_{k} \partial_{l} g_{i j}^{\prime}+g^{\prime k l} g_{i j}^{\prime} \partial_{k} \partial_{l} g^{i j}+J_{q}{ }^{p} g^{\prime k l}\left(\nabla_{k} J_{l}^{q}\right) g^{i j} \partial_{p} g_{i j}^{\prime} .
$$

The first terms on the right hand side of (3.2) and (3.3) are related as follows:

$$
\begin{aligned}
g^{\prime k l} g^{i j} \partial_{i} \partial_{j} g_{k l}^{\prime}= & g^{\prime k l} g^{i j} \partial_{k} \partial_{l} g_{i j}^{\prime}-J_{r}{ }^{k} g^{i j} \partial_{i} \partial_{j} J_{k}{ }^{r}-2 J_{j r}^{\prime} g^{\prime k l} g^{i j} \partial_{i} \partial_{l} J_{k}{ }^{r} \\
& +J_{r j}^{\prime} g^{\prime k l} g^{i j} \partial_{k} \partial_{l} J_{i}{ }^{r}-2 J_{r}{ }^{k} g^{i j} \partial_{k} \partial_{j} J_{i}{ }^{r} \\
& -2 g^{\prime k l} g^{i j}\left(\partial_{i} J_{k}{ }^{r}+\partial_{k} J_{i}{ }^{r}\right) J_{j}{ }^{q} \partial_{r} g_{q l}^{\prime} \\
& -2 g^{i j}\left(\partial_{i} J_{q}{ }^{r}+\partial_{q} J_{i}{ }^{r}\right) \partial_{r} J_{j}{ }^{q} \\
& -2 g^{\prime k l} g^{i j}\left(\partial_{l} J_{k}{ }^{r}\right) J_{j}{ }^{s} \partial_{i} g_{r s}^{\prime}
\end{aligned}
$$

To see this, calculate

$$
\begin{aligned}
g^{\prime k l} g^{i j} \partial_{i} \partial_{j} g_{k l}^{\prime}= & -g^{\prime k l} g^{i j} \partial_{i} \partial_{j}\left(J_{k}{ }^{r} J_{r l}^{\prime}\right) \\
= & -J_{k}{ }^{r} g^{\prime k l} g^{i j} \partial_{i} \partial_{j} J_{r l}^{\prime}-J_{r l}^{\prime} g^{\prime k l} g^{i j} \partial_{i} \partial_{j} J_{k}{ }^{r} \\
& -2 g^{\prime k l} g^{i j}\left(\partial_{i} J_{k}{ }^{r}\right)\left(\partial_{j} J_{r l}^{\prime}\right) .
\end{aligned}
$$

We will now apply (2.1) to the first term on the right hand side of the above equation to obtain

$$
\begin{aligned}
-J_{k}{ }^{r} g^{\prime k l} g^{i j} \partial_{i} \partial_{j} J_{r l}^{\prime}= & -J_{k}^{r} g^{\prime k l} g^{i j} \partial_{i}\left(\partial_{r} J_{j l}^{\prime}+\partial_{l} J_{r j}^{\prime}\right) \\
= & J^{\prime l r} g^{i j}\left(\partial_{i} \partial_{l} J_{j r}^{\prime}+\partial_{i} \partial_{l} J_{j r}^{\prime}\right) \\
= & 2 J_{k}{ }^{r} g^{\prime k l} g^{i j} \partial_{i} \partial_{l} J_{j r}^{\prime} \\
= & 2 g^{\prime k l} g^{i j} \partial_{i} \partial_{l}\left(J_{k}{ }^{r} J_{j r}^{\prime}\right)-2 g^{\prime k l} g^{i j}\left(\partial_{i} J_{k}{ }^{r}\right)\left(\partial_{l} J_{j r}^{\prime}\right) \\
& -2 g^{\prime k l} g^{i j}\left(\partial_{l} J_{k}{ }^{r}\right)\left(\partial_{i} J_{j r}^{\prime}\right)-2 J_{j r}^{\prime} g^{\prime k l} g^{i j} \partial_{i} \partial_{l} J_{k}{ }^{r} .
\end{aligned}
$$

Then in (3.5) we have

$$
\begin{aligned}
g^{\prime k l} g^{i j} \partial_{i} \partial_{j} g_{k l}^{\prime}= & 2 g^{\prime k l} g^{i j} \partial_{i} \partial_{l} g_{k j}^{\prime}-2 g^{\prime k l} g^{i j}\left(\partial_{i} J_{k}{ }^{r}\right)\left(\partial_{l} J_{j r}^{\prime}\right) \\
& -2 g^{\prime k l} g^{i j}\left(\partial_{l} J_{k}{ }^{r}\right)\left(\partial_{i} J_{j r}^{\prime}\right)-2 J_{j r}^{\prime} g^{\prime k l} g^{i j} \partial_{i} \partial_{l} J_{k}{ }^{r} \\
& -J_{r l}^{\prime} g^{\prime k l} g^{i j} \partial_{i} \partial_{j} J_{k}{ }^{r}-2 g^{\prime k l} g^{i j}\left(\partial_{i} J_{k}{ }^{r}\right)\left(\partial_{j} J_{r l}^{\prime}\right) .
\end{aligned}
$$


Similarly,

$$
\begin{aligned}
g^{\prime k l} g^{i j} \partial_{k} \partial_{l} g_{i j}^{\prime}= & 2 g^{i j} g^{\prime k l} \partial_{k} \partial_{j} g_{i l}^{\prime}-2 g^{i j} g^{\prime k l}\left(\partial_{k} J_{i}{ }^{r}\right)\left(\partial_{j} J_{l r}^{\prime}\right) \\
& -2 g^{i j} g^{\prime k l}\left(\partial_{j} J_{i}{ }^{r}\right)\left(\partial_{k} J_{l r}^{\prime}\right)-2 J_{l r}^{\prime} g^{i j} g^{\prime k l} \partial_{k} \partial_{j} J_{i}{ }^{r} \\
& -J_{r j}^{\prime} g^{\prime k l} g^{i j} \partial_{k} \partial_{l} J_{i}{ }^{r}-2 g^{\prime k l} g^{i j}\left(\partial_{k} J_{i}{ }^{r}\right)\left(\partial_{l} J_{r j}^{\prime}\right) .
\end{aligned}
$$

Combining (3.6) and (3.7) and again making use of (2.1) gives (3.4). From (3.2), (3.3) and (3.4) we obtain

$$
\begin{aligned}
\Delta^{\prime}\left(\log \operatorname{tr}_{g} g^{\prime}\right)= & \frac{1}{\operatorname{tr}_{g} g^{\prime}}\left(\Delta^{\prime}\left(\operatorname{tr}_{g} g^{\prime}\right)-\frac{\left|\nabla^{\prime} \operatorname{tr}_{g} g^{\prime}\right|^{2}}{\operatorname{tr}_{g} g^{\prime}}\right) \\
= & \frac{1}{\operatorname{tr}_{g} g^{\prime}}\left(2 \Delta F+g^{\prime k l} g^{i j} g^{p q}\left(\nabla_{p} g_{i k}^{\prime}\right)\left(\nabla_{q} g_{j l}^{\prime}\right)\right. \\
& +2 g^{\prime k l} g^{i j}\left(\nabla_{i} J_{k}{ }^{r}+\nabla_{k} J_{i}^{r}\right) J_{j}{ }^{q}\left(\nabla_{r} g_{q l}^{\prime}\right) \\
& +2 g^{i j}\left(\nabla_{i} J_{q}{ }^{r}+\nabla_{q} J_{i}{ }^{r}\right)\left(\nabla_{r} J_{j}{ }^{q}\right) \\
& +g^{\prime k l}\left(\nabla_{k} J_{l}{ }^{r}\right) g^{i j}\left(2 J_{j}{ }^{s} \nabla_{i} g_{r s}^{\prime}+J_{r}{ }^{s} \nabla_{s} g_{i j}^{\prime}\right) \\
& +J_{r}{ }^{k} g^{i j} \nabla_{i} \nabla_{j} J_{k}{ }^{r}-2 g^{i j} g^{\prime k l} g_{q j}^{\prime} J_{r}{ }^{q} \nabla_{i} \nabla_{l} J_{k}{ }^{r} \\
& -J_{r}{ }^{q} g_{q j}^{\prime} g^{\prime k l} g^{i j} \nabla_{k} \nabla_{l} J_{i}{ }^{r}-2 R \\
& \left.+2 g^{\prime k l} g_{i j}^{\prime} g^{p j} R_{l p k}^{i}-\frac{\left|\nabla^{\prime} \operatorname{tr}_{g} g^{\prime}\right|^{2}}{\operatorname{tr}_{g} g^{\prime}}\right),
\end{aligned}
$$

where, by definition,

$$
\left|\nabla^{\prime} \operatorname{tr}_{g} g^{\prime}\right|^{2}=g^{\prime k l}\left(\partial_{k} \operatorname{tr}_{g} g^{\prime}\right)\left(\partial_{l} \operatorname{tr}_{g} g^{\prime}\right),
$$

and where we are making use of the equations

$$
g^{i j} g^{k l} \partial_{i} \partial_{j} g_{k l}+2 J_{r}{ }^{k} g^{i j} \partial_{k} \partial_{j} J_{i}{ }^{r}=-2 R
$$

and

$$
\begin{aligned}
& 2 J_{j r}^{\prime} g^{\prime k l} g^{i j} \partial_{i} \partial_{l} J_{k}{ }^{r}+g^{\prime k l} g_{i j}^{\prime} \partial_{k} \partial_{l} g^{i j} \\
& =-2 g^{i j} g^{\prime k l} g_{q j}^{\prime} J_{r}{ }^{q} \nabla_{i} \nabla_{l} J_{k}{ }^{r}+2 g^{\prime k l} g_{i j}^{\prime} g^{p j} R_{l p k}^{i},
\end{aligned}
$$

which both hold only at a point.

Notice now that (3.8) is an equation of tensors. Since we are going to apply the maximum principle we need to obtain a good lower bound for the right hand side of (3.8). We have to deal with the bad terms that involve derivatives of $g^{\prime}$ and are not nonnegative: namely, the third, fifth and last terms. First, we need a lemma. 
Lemma 3.1. Define tensors $\alpha_{i j p}$ and $\beta_{i j p}$ by

$$
\begin{aligned}
\alpha_{i j p}= & J_{p l}^{\prime}\left(\nabla_{i} J_{j}{ }^{l}-\nabla_{j} J_{i}{ }^{l}\right)+\left(\nabla_{p} J_{l}{ }^{k}\right)\left(g_{k j}^{\prime} J_{i}{ }^{l}-g_{k i}^{\prime} J_{j}{ }^{l}\right) \\
& +g_{k p}^{\prime}\left(\left(\nabla_{l} J_{j}{ }^{k}\right) J_{i}{ }^{l}-\left(\nabla_{l} J_{i}{ }^{k}\right) J_{j}{ }^{l}\right), \\
\beta_{i j p}= & g_{k l}^{\prime}\left(\left(\nabla_{j} J_{i}{ }^{l}\right) J_{p}{ }^{k}-\left(\nabla_{j} J_{p}{ }^{l}\right) J_{i}{ }^{k}\right)-g_{k j}^{\prime}\left(\left(\nabla_{p} J_{l}{ }^{k}\right) J_{i}{ }^{l}-\left(\nabla_{i} J_{l}{ }^{k}\right) J_{p}{ }^{l}\right) \\
& -\left(\nabla_{l} J_{j}{ }^{k}\right)\left(g_{k p}^{\prime} J_{i}{ }^{l}-g_{k i}^{\prime} J_{p}{ }^{l}\right) .
\end{aligned}
$$

Then

(i) $2 \mathcal{P}_{i j}^{r s} \nabla_{r} g_{s p}^{\prime}-2 \mathcal{P}_{j i}^{r s} \nabla_{r} g_{s p}^{\prime}=\alpha_{i j p}$,

(ii) $2 \mathcal{Q}_{i j}^{r s} \nabla_{r} g_{s p}^{\prime}-2 \mathcal{Q}_{p j}^{r s} \nabla_{r} g_{s i}^{\prime}=\beta_{i j p}$.

Notice that in the Kähler case the tensors $\alpha$ and $\beta$ vanish identically and the lemma states that $\mathcal{P}_{i j}^{r s} \nabla_{r} g_{s p}^{\prime}$ is symmetric in $i$ and $j$ while $\mathcal{Q}_{i j}^{r s} \nabla_{r} g_{s p}^{\prime}$ is symmetric in $i$ and $p$. It is important here that $\alpha$ and $\beta$ do not contain derivatives of $g^{\prime}$.

Proof. We prove (i). The proof of (ii) is similar. From

$$
\nabla_{i} J_{j k}^{\prime}+\nabla_{j} J_{k i}^{\prime}+\nabla_{k} J_{i j}^{\prime}=0
$$

we have

$J_{j}{ }^{q} \nabla_{i} g_{q k}^{\prime}+J_{k}{ }^{q} \nabla_{j} g_{q i}^{\prime}+J_{i}{ }^{q} \nabla_{k} g_{q j}^{\prime}+g_{q k}^{\prime} \nabla_{i} J_{j}{ }^{q}+g_{q i}^{\prime} \nabla_{j} J_{k}{ }^{q}+g_{q j}^{\prime} \nabla_{k} J_{i}{ }^{q}=0$.

Multiplying by $J_{p}^{j}$ we obtain

$$
\begin{aligned}
\nabla_{i} g_{p k}^{\prime}= & J_{p}{ }^{j} J_{k}{ }^{q} \nabla_{j} g_{q i}^{\prime}+J_{p}{ }^{j} J_{i}{ }^{q} \nabla_{k} g_{j q}^{\prime}+g_{q k}^{\prime} J_{p}{ }^{j} \nabla_{i} J_{j}{ }^{q} \\
& +g_{q i}^{\prime} J_{p}{ }^{j} \nabla_{j} J_{k}{ }^{q}+g_{q j}^{\prime} J_{p}{ }^{j} \nabla_{k} J_{i}{ }^{q} \\
= & -2 \mathcal{P}_{p k}^{r s} \nabla_{r} g_{s i}^{\prime}+\nabla_{p} g_{k i}^{\prime}+\nabla_{k} g_{p i}^{\prime}-g_{q j}^{\prime} J_{i}{ }^{q} \nabla_{k} J_{p}{ }^{j} \\
& +g_{q k}^{\prime} J_{p}{ }^{j} \nabla_{i} J_{j}{ }^{q}+g_{q i}^{\prime} J_{p}{ }^{j} \nabla_{j} J_{k}{ }^{q},
\end{aligned}
$$

where we have made use of the identity $\mathcal{P}_{p i}^{j q} g_{j q}^{\prime}=0$. Then (i) follows easily.

q.e.d.

We return to equation (3.8). The third term on the right hand side (ignoring the factor of $1 / \operatorname{tr}_{g} g^{\prime}$ ) can be written

$$
\begin{aligned}
& 2 g^{\prime k l} g^{i j}\left(\nabla_{i} J_{k}{ }^{r}+\nabla_{k} J_{i}{ }^{r}\right) J_{j}{ }^{q} \nabla_{r} g_{q l}^{\prime} \\
& =2 g^{\prime k l} g^{i j} g^{r s}\left(\nabla_{i} J_{k r}+\nabla_{k} J_{i r}\right) \nabla_{s} J_{j l}^{\prime} \\
& \quad-2 g^{\prime k l} g^{i j} g^{r s}\left(\nabla_{i} J_{k r}+\nabla_{k} J_{i r}\right) g_{q l}^{\prime} \nabla_{s} J_{j}{ }^{q} .
\end{aligned}
$$


Making use of the identity $\mathcal{Q}_{i r}^{a b} \nabla_{k} J_{a b}=0$ we rewrite the first term on the right hand side of (3.9) as

$$
\begin{aligned}
& 2 g^{\prime k l} g^{i j} g^{r s}\left(\nabla_{i} J_{k r}+\nabla_{k} J_{i r}\right) \nabla_{s} J_{j l}^{\prime} \\
& =4 g^{\prime k l} g^{i j} g^{r s}\left(\nabla_{k} J_{i r}\right)\left(\nabla_{s} J_{j l}^{\prime}\right)+2 g^{\prime k l} g^{i j} g^{r s}\left(\nabla_{r} J_{k i}\right)\left(\nabla_{s} J_{j l}^{\prime}\right) \\
& =4 g^{\prime k l} g^{i j} g^{r s} \mathcal{P}_{i r}^{a b}\left(\nabla_{k} J_{a b}\right)\left(\nabla_{s} J_{j l}^{\prime}\right) \\
& \quad+2 g^{\prime k l} g^{i j} g^{r s} \mathcal{P}_{k i}^{a b}\left(\nabla_{r} J_{a b}\right)\left(\nabla_{s} J_{j l}^{\prime}\right) \\
& =(I)+(I I),
\end{aligned}
$$

where

$$
(I)=4 g^{\prime k l} g^{i j} g^{r s}\left(\nabla_{k} J_{i r}\right) \mathcal{P}_{j s}^{a b}\left(\nabla_{b} J_{a l}^{\prime}\right)
$$

and

$$
(I I)=2 g^{\prime k l} g^{i j} g^{r s}\left(\nabla_{r} J_{i k}\right) \mathcal{P}_{j l}^{a b}\left(\nabla_{s} J_{a b}^{\prime}\right) .
$$

To deal with $(I)$, first note that

$$
\begin{aligned}
\mathcal{P}_{j s}^{a b} \nabla_{b} J_{a l}^{\prime} & =\mathcal{P}_{s j}^{a b} \nabla_{a} J_{b l}^{\prime} \\
& =-\mathcal{P}_{s j}^{a b}\left(\nabla_{a} g_{b q}^{\prime}\right) J_{l}{ }^{q}-\mathcal{P}_{s j}^{a b} g_{b q}^{\prime} \nabla_{a} J_{l}{ }^{q} \\
& =-\mathcal{P}_{j s}^{a b}\left(\nabla_{a} g_{b q}^{\prime}\right) J_{l}{ }^{q}-\frac{1}{2} \alpha_{s j q} J_{l}{ }^{q}-\mathcal{P}_{s j}^{a b} g_{b q}^{\prime} \nabla_{a} J_{l}{ }^{q} \\
& =\mathcal{P}_{s j}^{a b} \nabla_{b} J_{a l}^{\prime}+\mathcal{P}_{j s}^{a b} g_{b q}^{\prime} \nabla_{a} J_{l}{ }^{q}-\frac{1}{2} \alpha_{s j q} J_{l}{ }^{q}-\mathcal{P}_{s j}^{a b} g_{b q}^{\prime} \nabla_{a} J_{l}{ }^{q} .
\end{aligned}
$$

That is, the term $\mathcal{P}_{j s}^{a b} \nabla_{b} J_{a l}^{\prime}$ is symmetric in $j$ and $s$ modulo terms that don't involve derivatives of $g^{\prime}$. Then

$$
\begin{aligned}
(I)= & -4 g^{\prime k l} g^{i j} g^{r s}\left(\nabla_{k} J_{r i}\right) \mathcal{P}_{s j}^{a b} \nabla_{b} J_{a l}^{\prime} \\
& +4 g^{\prime k l} g^{i j} g^{r s}\left(\nabla_{k} J_{i r}\right)\left(\mathcal{P}_{j s}^{a b} g_{b q}^{\prime} \nabla_{a} J_{l}^{q}-\frac{1}{2} \alpha_{s j q} J_{l}^{q}-\mathcal{P}_{s j}^{a b} g_{b q}^{\prime} \nabla_{a} J_{l}^{q}\right),
\end{aligned}
$$

and hence, interchanging the indices $i$ and $j$ with $r$ and $s$ in the first term, we have

$$
\begin{aligned}
(I)= & 2 g^{\prime k l} g^{i j} g^{r s}\left(\nabla_{k} J_{i r}\right)\left(\mathcal{P}_{j s}^{a b} g_{b q}^{\prime} \nabla_{a} J_{l}^{q}\right. \\
& \left.-\frac{1}{2} \alpha_{s j q} J_{l}^{q}-\mathcal{P}_{s j}^{a b} g_{b q}^{\prime} \nabla_{a} J_{l}^{q}\right) .
\end{aligned}
$$

For $(I I)$, calculate

$$
\begin{aligned}
\mathcal{P}_{j l}^{a b} \nabla_{s} J_{a b}^{\prime} & =\frac{1}{2}\left(\nabla_{s} J_{j l}^{\prime}-J_{j}{ }^{a} J_{l}{ }^{b} \nabla_{s} J_{a b}^{\prime}\right) \\
& =\frac{1}{2}\left(\nabla_{s} J_{j l}^{\prime}-\nabla_{s} J_{j l}^{\prime}+J_{a b}^{\prime} J_{l}{ }^{b} \nabla_{s} J_{j}{ }^{a}+J_{a b}^{\prime} J_{j}{ }^{a} \nabla_{s} J_{l}{ }^{b}\right) \\
& =\frac{1}{2} J_{a b}^{\prime}\left(J_{l}{ }^{b} \nabla_{s}{J_{j}}^{a}+{J_{j}}^{a} \nabla_{s} J_{l}{ }^{b}\right)
\end{aligned}
$$


Then from equations (3.9), (3.10), (3.11) and (3.12) we have the following expression for the third term of (3.8):

$$
\begin{aligned}
& 2 g^{\prime k l} g^{i j}\left(\nabla_{i} J_{k}{ }^{r}+\nabla_{k} J_{i}{ }^{r}\right) J_{j}{ }^{q} \nabla_{r} g_{q l}^{\prime} \\
& =2 g^{\prime k l} g^{i j} g^{r s}\left(\nabla_{k} J_{i r}\right)\left(\mathcal{P}_{j s}^{a b} g_{b q}^{\prime} \nabla_{a} J_{l}{ }^{q}-\frac{1}{2} \alpha_{s j q} J_{l}{ }^{q}-\mathcal{P}_{s j}^{a b} g_{b q}^{\prime} \nabla_{a} J_{l}{ }^{q}\right) \\
& \quad+g^{\prime k l} g^{i j} g^{r s}\left(\nabla_{r} J_{i k}\right) J_{a b}^{\prime}\left(J_{l}{ }^{b} \nabla_{s} J_{j}{ }^{a}+J_{j}{ }^{a} \nabla_{s} J_{l}{ }^{b}\right) \\
& \quad-2 g^{\prime k l} g^{i j} g^{r s}\left(\nabla_{i} J_{k r}+\nabla_{k} J_{i r}\right) g_{q l}^{\prime} \nabla_{s} J_{j}{ }^{q} .
\end{aligned}
$$

We deal now with the fifth term on the right hand side of (3.8). Calculate:

$$
\begin{aligned}
& g^{\prime k l}\left(\nabla_{k} J_{l}{ }^{r}\right) g^{i j}\left(2 J_{j}{ }^{s} \nabla_{i} g_{r s}^{\prime}+J_{r}{ }^{s} \nabla_{s} g_{i j}^{\prime}\right) \\
&=g^{\prime k l}\left(\nabla_{k} J_{l}{ }^{r}\right) g^{i j}\left(2 \nabla_{i} J_{j r}^{\prime}-J_{r}{ }^{s} \nabla_{s}\left(J_{p j}^{\prime} J_{i}{ }^{p}\right)\right) \\
&=g^{\prime k l}\left(\nabla_{k} J_{l}{ }^{r}\right) g^{i j}\left(2 \nabla_{i} J_{j r}^{\prime}-J_{r}{ }^{s} J_{i}{ }^{p} \nabla_{p} J_{s j}^{\prime}\right. \\
&\left.-J_{r}{ }^{s} J_{i}{ }^{p} \nabla_{j} J_{p s}^{\prime}-J_{r}{ }^{s} J_{p j}^{\prime} \nabla_{s} J_{i}{ }^{p}\right) \\
&= g^{\prime k l}\left(\nabla_{k} J_{l}{ }^{r}\right) g^{i j}\left(2 \nabla_{i} J_{j r}^{\prime}+J_{i}{ }^{a} J_{r}{ }^{b} \nabla_{a} J_{j b}^{\prime}-\nabla_{j} J_{i r}^{\prime}\right. \\
&\left.+J_{i}{ }^{p} J_{p s}^{\prime} \nabla_{j} J_{r}{ }^{s}-J_{r}{ }^{s} J_{p j}^{\prime} \nabla_{s} J_{i}{ }^{p}\right) \\
&= g^{\prime k l}\left(\nabla_{k} J_{l}{ }^{r}\right) g^{i j}\left(2 \mathcal{Q}_{i r}^{a b} \nabla_{a} J_{j b}^{\prime}+J_{i}{ }^{p} J_{p s}^{\prime} \nabla_{j} J_{r}{ }^{s}-J_{r}{ }^{s} J_{p j}^{\prime} \nabla_{s} J_{i}{ }^{p}\right) \\
&= g^{\prime k l}\left(\nabla_{k} J_{l}{ }^{r}\right) g^{i j}\left(2 \mathcal{Q}_{i r}^{a b}\left(\nabla_{a} g_{b q}^{\prime}\right) J_{j}{ }^{q}+2 \mathcal{Q}_{i r}^{a b} g_{b q}^{\prime} \nabla_{a} J_{j}{ }^{q}-g_{i s}^{\prime} \nabla_{j} J_{r}{ }^{i}\right. \\
&\left.-J_{r}{ }^{s} J_{p j}^{\prime} \nabla_{s} J_{i}{ }^{p}\right) \\
&= g^{\prime k l}\left(\nabla_{k} J_{l}{ }^{r}\right)\left(\frac{1}{2} \beta_{i r q} J^{i q}+2 g^{i j} \mathcal{Q}_{i r}^{a b} g_{b q}^{\prime} \nabla_{a} J_{j}{ }^{q}-g^{i j} g_{i s}^{\prime} \nabla_{j} J_{r}{ }^{s}\right. \\
&\left.-g^{i j} J_{r}{ }^{s} J_{p j}^{\prime} \nabla_{s} J_{i}{ }^{p}\right),
\end{aligned}
$$

where, for the last line, we have used the identity

$$
2 \mathcal{Q}_{i r}^{a b}\left(\nabla_{a} g_{b q}^{\prime}\right) J^{i q}=\frac{1}{2} \beta_{i r q} J^{i q}
$$

which follows immediately from Lemma 3.1.

Finally, we must deal with the last term on the right hand side of (3.8). We do this by making use of the good second term.

Lemma 3.2. There exists a constant $C^{\prime}$ depending only on $\sup _{M}|F|$ and $\|\operatorname{Rm}(g)\|_{C^{0}(g)}$ such that

$$
\frac{\left|\nabla^{\prime}\left(\operatorname{tr}_{g} g^{\prime}\right)\right|^{2}}{\operatorname{tr}_{g} g^{\prime}} \leq g^{\prime k l} g^{i j} g^{p q}\left(\nabla_{p} g_{i k}^{\prime}\right)\left(\nabla_{q} g_{j l}^{\prime}\right)+C^{\prime}\left(\operatorname{tr}_{g} g^{\prime}\right)\left(\operatorname{tr}_{g^{\prime}} g\right) \text {. }
$$


To prove this lemma we will work in a coordinate system $\left(x^{1}, \ldots, x^{4}\right)$ centred at a point $p$ such that the first derivatives of the metric $g$ vanish at $p$ and

$$
J_{1}{ }^{3}=J_{2}{ }^{4}=1=-J_{3}{ }^{1}=-J_{4}{ }^{2},
$$

and all other entries of the matrix $\left(J_{i}{ }^{j}\right)$ are zero at $p$. Define local vector fields

$$
Z_{\alpha}=\frac{1}{2}\left(\partial_{\alpha}-\sqrt{-1} J_{\alpha}^{i} \partial_{i}\right)
$$

for $\alpha=1,2$. Set

$$
G_{\alpha \bar{\beta}}=g\left(Z_{\alpha}, \bar{Z}_{\beta}\right)
$$

and

$$
G_{\alpha \bar{\beta}}^{\prime}=g^{\prime}\left(Z_{\alpha}, \bar{Z}_{\beta}\right)
$$

for $\alpha, \beta=1,2$. Then we make a linear change in the coordinates $\left(x^{1}, \ldots, x^{4}\right)$ so that, in addition to the above conditions at $p$, we also impose that $G_{\alpha \bar{\beta}}=\delta_{\alpha \beta}$ and that $G_{\alpha \bar{\beta}}^{\prime}$ be diagonal. Notice that in these coordinates, the first derivatives of the $g_{i j}$ vanish at $p$, but in general, the first derivatives of the $G_{\alpha \bar{\beta}}$ will not.

It will also be useful to consider the local vector fields

$$
W_{\alpha}=\frac{1}{2}\left(-J_{A}^{i} \partial_{i}-\sqrt{-1} \partial_{A}\right),
$$

for $\alpha=1,2$, where we are setting $A=\alpha+2$. Notice that, at $p$, $W_{\alpha}=Z_{\alpha}$. In the sequel, we will use the indices $A, B, C, D, M, N$ to denote $\alpha+2, \beta+2, \gamma+2, \delta+2, \mu+2, \nu+2$. Set

$$
\tilde{G}_{\alpha \bar{\beta}}=g\left(W_{\alpha}, \bar{W}_{\beta}\right)
$$

and

$$
\tilde{G}_{\alpha \bar{\beta}}^{\prime}=g^{\prime}\left(W_{\alpha}, \bar{W}_{\beta}\right)
$$

Observe that

$$
G_{\alpha \bar{\beta}}=\frac{1}{2}\left(g_{\alpha \beta}-\sqrt{-1} J_{\alpha \beta}\right), \quad G_{\alpha \bar{\beta}}^{\prime}=\frac{1}{2}\left(g_{\alpha \beta}^{\prime}-\sqrt{-1} J_{\alpha \beta}^{\prime}\right),
$$

and

$$
\tilde{G}_{\alpha \bar{\beta}}=\frac{1}{2}\left(g_{A B}-\sqrt{-1} J_{A B}\right), \quad \tilde{G}_{\alpha \bar{\beta}}^{\prime}=\frac{1}{2}\left(g_{A B}^{\prime}-\sqrt{-1} J_{A B}^{\prime}\right) .
$$

At the point $p, G=\tilde{G}$ and $G^{\prime}=\tilde{G}^{\prime}$; this fact will be used later in the proof of Lemma 3.2. Notice also that $g_{i j}=2 \delta_{i j}$ and that $g_{i j}^{\prime}$ is diagonal. A final word about notation: when we are using the local vector fields $Z_{\alpha}$ and $\bar{Z}_{\alpha}$ as differential operators, we will instead write $D_{\alpha}$ and $D_{\bar{\alpha}}$ respectively. We require some preliminary results before we prove Lemma 3.2. 


\section{Lemma 3.3.}

$$
\begin{aligned}
D_{\gamma} G_{\alpha \bar{\beta}}^{\prime} & =D_{\alpha} G_{\gamma \bar{\beta}}^{\prime}+\frac{1}{4}\left(a_{\gamma \alpha \beta}+\sqrt{-1} b_{\gamma \alpha \beta}\right) \\
\text { and } \quad D_{\gamma} \tilde{G}_{\alpha \bar{\beta}}^{\prime} & =D_{\alpha} \tilde{G}_{\gamma \bar{\beta}}^{\prime}+\frac{1}{4}\left(a_{\gamma A B}+\sqrt{-1} b_{\gamma A B}\right),
\end{aligned}
$$

where

$$
\begin{aligned}
a_{\gamma i j} & =\left(\partial_{i} J_{\gamma}{ }^{k}-\partial_{\gamma} J_{i}{ }^{k}\right) J_{k j}^{\prime}+\left(\partial_{j} J_{\gamma}{ }^{k}\right) J_{i k}^{\prime}-\left(\partial_{j} J_{i}{ }^{k}\right) J_{\gamma k}^{\prime} \\
\text { and } \quad b_{\gamma i j} & =g_{k i}^{\prime} \partial_{j} J_{\gamma}{ }^{k}-g_{k \gamma}^{\prime} \partial_{j} J_{i}{ }^{k}+J_{j l}^{\prime}\left(J_{i}{ }^{k} \partial_{k} J_{\gamma}{ }^{l}-J_{\gamma}{ }^{k} \partial_{k} J_{i}{ }^{l}\right) .
\end{aligned}
$$

Proof. We will just prove (3.15), since the proof of (3.16) is similar. Calculate

$$
\begin{aligned}
& D_{\gamma} G_{\alpha \bar{\beta}}^{\prime}=\frac{1}{4}\left(\partial_{\gamma}-\sqrt{-1} J_{\gamma}{ }^{i} \partial_{i}\right)\left(g_{\alpha \beta}^{\prime}-\sqrt{-1} J_{\alpha \beta}^{\prime}\right) \\
& =\frac{1}{4}\left(\partial_{\gamma} g_{\alpha \beta}^{\prime}-J_{\gamma}{ }^{i} \partial_{i} J_{\alpha \beta}^{\prime}-\sqrt{-1} J_{\gamma}{ }^{i} \partial_{i} g_{\alpha \beta}^{\prime}-\sqrt{-1} \partial_{\gamma} J_{\alpha \beta}^{\prime}\right) \\
& =\frac{1}{4}\left(\partial_{\gamma} g_{\alpha \beta}^{\prime}-J_{\gamma}{ }^{i} \partial_{\alpha} J_{i \beta}^{\prime}-J_{\gamma}{ }^{i} \partial_{\beta} J_{\alpha i}^{\prime}-\sqrt{-1} J_{\gamma}{ }^{i} \partial_{i} g_{\alpha \beta}^{\prime}\right. \\
& \left.-\sqrt{-1} \partial_{\alpha} J_{\gamma \beta}^{\prime}-\sqrt{-1} \partial_{\beta} J_{\alpha \gamma}^{\prime}\right) \\
& =\frac{1}{4}\left(\partial_{\gamma} g_{\alpha \beta}^{\prime}+\partial_{\alpha} g_{\gamma \beta}^{\prime}+\left(\partial_{\alpha} J_{\gamma}{ }^{i}\right) J_{i \beta}^{\prime}-J_{\alpha}{ }^{i} \partial_{\beta} J_{\gamma i}^{\prime}-\left(\partial_{\beta} J_{\alpha}{ }^{i}\right) J_{\gamma i}^{\prime}\right. \\
& +\left(\partial_{\beta} J_{\gamma}{ }^{i}\right) J_{\alpha i}^{\prime}-\sqrt{-1} J_{\gamma}{ }^{i} \partial_{i}\left(J_{\beta p}^{\prime} J_{\alpha}{ }^{p}\right)-\sqrt{-1} \partial_{\alpha} J_{\gamma \beta}^{\prime} \\
& \left.-\sqrt{-1} \partial_{\beta} J_{\alpha \gamma}^{\prime}\right) \\
& =\frac{1}{4}\left(\partial_{\gamma} g_{\alpha \beta}^{\prime}+\partial_{\alpha} g_{\gamma \beta}^{\prime}+\left(\partial_{\alpha} J_{\gamma}{ }^{i}\right) J_{i \beta}^{\prime}-J_{\alpha}{ }^{i} \partial_{i} J_{\gamma \beta}^{\prime}-J_{\alpha}{ }^{i} \partial_{\gamma} J_{\beta i}^{\prime}\right. \\
& -\left(\partial_{\beta} J_{\alpha}{ }^{i}\right) J_{\gamma i}^{\prime}+\left(\partial_{\beta} J_{\gamma}{ }^{i}\right) J_{\alpha i}^{\prime}-\sqrt{-1} J_{\gamma}{ }^{i} J_{\alpha}{ }^{p} \partial_{i} J_{\beta p}^{\prime} \\
& \left.-\sqrt{-1} J_{\gamma}{ }^{i} J_{\beta p}^{\prime} \partial_{i} J_{\alpha}{ }^{p}-\sqrt{-1} \partial_{\alpha} J_{\gamma \beta}^{\prime}-\sqrt{-1} \partial_{\beta} J_{\alpha \gamma}^{\prime}\right),
\end{aligned}
$$

where to go from the third to the fourth lines we have used the simple identity

$$
J_{\gamma}{ }^{i} \partial_{\beta} J_{\alpha i}^{\prime}+\left(\partial_{\beta} J_{\gamma}{ }^{i}\right) J_{\alpha i}^{\prime}=\left(\partial_{\beta} J_{\alpha}{ }^{i}\right) J_{\gamma i}^{\prime}+J_{\alpha}{ }^{i} \partial_{\beta} J_{\gamma i}^{\prime} .
$$

Notice that

$$
\begin{aligned}
J_{\gamma}{ }^{i} J_{\alpha}{ }^{p} \partial_{i} J_{\beta p}^{\prime} & =J_{\gamma}{ }^{i} J_{\alpha}{ }^{p} \partial_{\beta} J_{i p}^{\prime}+J_{\gamma}{ }^{i} J_{\alpha}{ }^{p} \partial_{p} J_{\beta i}^{\prime} \\
& =\partial_{\beta} J_{\gamma \alpha}^{\prime}-J_{i p}^{\prime} J_{\alpha}{ }^{p} \partial_{\beta} J_{\gamma}{ }^{i}-J_{i p}^{\prime} J_{\gamma}{ }^{i} \partial_{\beta} J_{\alpha}{ }^{p}+J_{\alpha}{ }^{i} J_{\gamma}{ }^{p} \partial_{i} J_{\beta p}^{\prime}
\end{aligned}
$$


In (3.17) this gives us

$$
\begin{aligned}
& D_{\gamma} G_{\alpha \bar{\beta}}^{\prime} \\
&=\frac{1}{4}\left(\partial_{\gamma} g_{\alpha \beta}^{\prime}+\partial_{\alpha} g_{\gamma \beta}^{\prime}+\left(\partial_{\alpha} J_{\gamma}{ }^{i}\right) J_{i \beta}^{\prime}-J_{\alpha}{ }^{i} \partial_{i} J_{\gamma \beta}^{\prime}-J_{\alpha}{ }^{i} \partial_{\gamma} J_{\beta i}^{\prime}\right. \\
& \quad-\left(\partial_{\beta} J_{\alpha}{ }^{i}\right) J_{\gamma i}^{\prime}+\left(\partial_{\beta} J_{\gamma}{ }^{i}\right) J_{\alpha i}^{\prime}+\sqrt{-1} J_{i p}^{\prime} J_{\alpha}{ }^{p} \partial_{\beta} J_{\gamma}{ }^{i}+\sqrt{-1} J_{i p}^{\prime} J_{\gamma}{ }^{i} \partial_{\beta} J_{\alpha}{ }^{p} \\
&\left.\quad-\sqrt{-1} J_{\alpha}{ }^{i} J_{\gamma}{ }^{p} \partial_{i} J_{\beta p}^{\prime}-\sqrt{-1} J_{\gamma}{ }^{i} J_{\beta p}^{\prime} \partial_{i} J_{\alpha}{ }^{p}-\sqrt{-1} \partial_{\alpha} J_{\gamma \beta}^{\prime}\right) \\
&=\frac{1}{4}\left(\partial_{\alpha} g_{\gamma \beta}^{\prime}+\left(\partial_{\alpha} J_{\gamma}{ }^{i}\right) J_{i \beta}^{\prime}-J_{\alpha}{ }^{i} \partial_{i} J_{\gamma \beta}^{\prime}+\left(\partial_{\gamma} J_{\alpha}{ }^{i}\right) J_{\beta i}^{\prime}-\left(\partial_{\beta} J_{\alpha}{ }^{i}\right) J_{\gamma i}^{\prime}\right. \\
&+\left(\partial_{\beta} J_{\gamma}{ }^{i}\right) J_{\alpha i}^{\prime}+\sqrt{-1} g_{i \alpha}^{\prime} \partial_{\beta} J_{\gamma}{ }^{i}-\sqrt{-1} g_{p \gamma}^{\prime} \partial_{\beta} J_{\alpha}{ }^{p}-\sqrt{-1} J_{\alpha}{ }^{i} \partial_{i} g_{\beta \gamma}^{\prime} \\
&\left.+\sqrt{-1} J_{\alpha}{ }^{i} J_{\beta p}^{\prime}\left(\partial_{i} J_{\gamma}{ }^{p}\right)-\sqrt{-1} J_{\gamma}{ }^{i} J_{\beta p}^{\prime}\left(\partial_{i} J_{\alpha}{ }^{p}\right)-\sqrt{-1} \partial_{\alpha} J_{\gamma \beta}^{\prime}\right) \\
&= D_{\alpha} G_{\gamma \bar{\beta}}^{\prime}+\frac{1}{4}\left(\left(\partial_{\alpha} J_{\gamma}{ }^{i}-\partial_{\gamma} J_{\alpha}{ }^{i}\right) J_{i \beta}^{\prime}-\left(\partial_{\beta} J_{\alpha}{ }^{i}\right) J_{\gamma i}^{\prime}+\left(\partial_{\beta} J_{\gamma}{ }^{i}\right) J_{\alpha i}^{\prime}\right. \\
&\left.+\sqrt{-1}\left(g_{i \alpha}^{\prime} \partial_{\beta} J_{\gamma}{ }^{i}-g_{i \gamma}^{\prime} \partial_{\beta} J_{\alpha}{ }^{i}\right)+\sqrt{-1} J_{\beta p}{ }\left(J_{\alpha}{ }^{i} \partial_{i} J_{\gamma}{ }^{p}-J_{\gamma}{ }^{i} \partial_{i} J_{\alpha}{ }^{p}\right)\right)
\end{aligned}
$$

as required.

q.e.d.

We will also need the following result.

Lemma 3.4. At the point $p$ we have

$$
\begin{aligned}
& 2 D_{\gamma}\left(G^{\alpha \bar{\beta}} G_{\alpha \bar{\beta}}^{\prime}\right)=2 G^{\alpha \bar{\beta}} D_{\gamma} G_{\alpha \bar{\beta}}^{\prime}=D_{\gamma}\left(g^{i j} g_{i j}^{\prime}\right)+E_{\gamma} \quad \text { and } \\
& 2 D_{\gamma}\left(\tilde{G}^{\alpha \bar{\beta}} \tilde{G}_{\alpha \bar{\beta}}^{\prime}\right)=2 \tilde{G}^{\alpha \bar{\beta}} D_{\gamma} \tilde{G}_{\alpha \bar{\beta}}^{\prime}=D_{\gamma}\left(g^{i j} g_{i j}^{\prime}\right)-E_{\gamma},
\end{aligned}
$$

where $E_{\gamma}=\sum_{i=1}^{2} g_{r s}^{\prime}\left(D_{\gamma} J_{i}{ }^{r}\right) J_{i}{ }^{s}$.

We are using here the usual notation for the inverse metrics $G^{-1}$ and $\tilde{G}^{-1}$. Note also that repeated greek indices $\alpha, \beta, \ldots$ are used to denote a sum from 1 to 2 , whereas repeated lower case roman letters $i, j, \ldots$ denote the usual sum from 1 to 4, unless otherwise indicated.

Proof. We will prove just (3.18). Calculate

$$
\begin{aligned}
2 D_{\gamma}\left(G^{\alpha \bar{\beta}} G_{\alpha \bar{\beta}}^{\prime}\right)= & -\frac{1}{2} G^{\alpha \bar{\mu}} G^{\nu \bar{\beta}}\left(-J_{\gamma}{ }^{i} \partial_{i} J_{\nu \mu}-\sqrt{-1} \partial_{\gamma} J_{\nu \mu}\right) G_{\alpha \bar{\beta}}^{\prime} \\
& +2 G^{\alpha \bar{\beta}} D_{\gamma} G_{\alpha \bar{\beta}}^{\prime} \\
= & 2 G^{\alpha \bar{\beta}} D_{\gamma} G_{\alpha \bar{\beta}}^{\prime} \\
= & \frac{1}{2} G^{\alpha \bar{\beta}}\left(\partial_{\gamma} g_{\alpha \beta}^{\prime}-\sqrt{-1} J_{\gamma}{ }^{k} \partial_{k} g_{\alpha \beta}^{\prime}\right) .
\end{aligned}
$$


But

$$
\begin{aligned}
D_{\gamma}\left(g^{i j} g_{i j}^{\prime}\right)= & \frac{1}{2} g^{i j}\left(\partial_{\gamma}-\sqrt{-1} J_{\gamma}{ }^{k} \partial_{k}\right) g_{i j}^{\prime} \\
= & \frac{1}{4} \sum_{i=1}^{4}\left(\partial_{\gamma} g_{i i}^{\prime}-\sqrt{-1} J_{\gamma}{ }^{k} \partial_{k} g_{i i}^{\prime}\right) \\
= & \frac{1}{2} \sum_{i=1}^{2}\left(\partial_{\gamma} g_{i i}^{\prime}-g_{r s}^{\prime}\left(\partial_{\gamma} J_{i}{ }^{r}\right) J_{i}{ }^{s}-\sqrt{-1} J_{\gamma}{ }^{k} \partial_{k} g_{i i}^{\prime}\right. \\
& \left.\quad+\sqrt{-1} J_{\gamma}{ }^{k} g_{r s}^{\prime}\left(\partial_{k} J_{i}{ }^{r}\right) J_{i}{ }^{s}\right),
\end{aligned}
$$

where we have used the fact that, at $p$,

$$
\partial_{k} g_{A B}^{\prime}-\partial_{k} g_{\alpha \beta}^{\prime}=-g_{r s}^{\prime}\left(\partial_{k} J_{\alpha}^{r}\right) J_{\beta}{ }^{s}-g_{r s}^{\prime}\left(\partial_{k} J_{\beta}{ }^{s}\right) J_{\alpha}{ }^{r} .
$$

Comparing (3.20) and (3.21) gives (3.18).

We need one more lemma before we can prove Lemma 3.2.

Lemma 3.5. At $p$,

$$
g^{i j} a_{\gamma i j}=0 \quad \text { and } \quad g^{i j} b_{\gamma i j}=0 .
$$

Proof. For the first equation, calculate at $p$ :

$$
\begin{aligned}
g^{i j} a_{\gamma i j}= & g^{i j}\left(\nabla_{i} J_{\gamma}{ }^{k}\right) J_{k j}^{\prime}-g^{i j}\left(\nabla_{\gamma} J_{i}{ }^{k}\right) J_{k j}^{\prime} \\
& +g^{i j}\left(\nabla_{j} J_{\gamma}{ }^{k}\right) J_{i k}^{\prime}-g^{i j}\left(\nabla_{j} J_{i}{ }^{k}\right) J_{\gamma k}^{\prime} \\
= & -g^{i j}\left(\nabla_{\gamma} J_{i}{ }^{k}\right) J_{k j}^{\prime} \\
= & -g^{i j}\left(\nabla_{\gamma} J_{i}{ }^{k}\right) J_{k}{ }^{s} g_{s j}^{\prime} \\
= & g^{i j} J_{i}{ }^{k}\left(\nabla_{\gamma} J_{k}{ }^{s}\right) g_{s j}^{\prime} \\
= & -g^{i k}{J_{i}}^{j}\left(\nabla_{\gamma} J_{k}{ }^{s}\right) g_{s j}^{\prime} \\
= & 0,
\end{aligned}
$$

by symmetry. For the second, calculate:

$$
\begin{aligned}
& g^{i j} b_{\gamma i j}=g^{i j} g_{k i}^{\prime}\left(\nabla_{j} J_{\gamma}{ }^{k}\right)-g^{i j} g_{k \gamma}^{\prime}\left(\nabla_{j} J_{i}{ }^{k}\right) \\
& +g^{i j} J_{j l}^{\prime} J_{i}{ }^{k}\left(\nabla_{k} J_{\gamma}{ }^{l}\right)-g^{i j} J_{j l}^{\prime} J_{\gamma}{ }^{k}\left(\nabla_{k} J_{i}{ }^{l}\right) \\
& =g^{i j} J_{k l}^{\prime} J_{i}{ }^{l}\left(\nabla_{j} J_{\gamma}{ }^{k}\right)-g^{i k} J_{j l}^{\prime} J_{i}{ }^{j}\left(\nabla_{k} J_{\gamma}{ }^{l}\right) \\
& -g^{i j} J_{j l}^{\prime} J_{\gamma}{ }^{k}\left(\nabla_{i} J_{k}{ }^{l}\right)-g^{i j} J_{j l}^{\prime} J_{\gamma}{ }^{k}\left(\nabla^{l} J_{i k}\right) \\
& =2 g^{i j} J_{k l}^{\prime} J_{i}{ }^{l}\left(\nabla_{j} J_{\gamma}{ }^{k}\right)-2 J_{\gamma}{ }^{k} J_{j l}^{\prime}\left(\nabla^{j} J_{k}{ }^{l}\right) .
\end{aligned}
$$

Notice that the second term vanishes since

$$
J_{j l}^{\prime} \nabla^{j} J_{k}^{l}=\frac{1}{2} g_{l p}^{\prime} J_{j}^{p} \nabla_{k} J^{j l}=0 .
$$


But also the first term can be written

$$
g^{i j} J_{k l}^{\prime} J_{i}{ }^{l}\left(\nabla_{j} J_{\gamma}{ }^{k}\right)=g^{i j} J_{k}{ }^{p} g_{p l}^{\prime} J_{i}{ }^{l}\left(\nabla_{j} J_{\gamma}{ }^{k}\right)=-J_{i p}^{\prime} J_{\gamma}{ }^{k}\left(\nabla^{i} J_{k}{ }^{p}\right)=0,
$$

finishing the proof of the lemma.

q.e.d.

Proof of Lemma 3.2.

Using Lemma 3.4 we have

$$
\begin{aligned}
g^{\prime k l} \partial_{k}\left(g^{i j} g_{i j}^{\prime}\right) \partial_{l}\left(g^{p q} g_{p q}^{\prime}\right) \\
=2 G^{\prime \gamma \bar{\delta}} D_{\gamma}\left(g^{i j} g_{i j}^{\prime}\right) D_{\bar{\delta}}\left(g^{p q} g_{p q}^{\prime}\right) \\
=G^{\prime \gamma \bar{\delta}}\left(2 D_{\gamma}\left(G^{\alpha \bar{\beta}} G_{\alpha \bar{\beta}}^{\prime}\right)-E_{\gamma}\right)\left(2 D_{\bar{\delta}}\left(G^{\mu \bar{\nu}} G_{\mu \bar{\nu}}^{\prime}\right)-\bar{E}_{\delta}\right) \\
\quad+G^{\prime \gamma \bar{\delta}}\left(2 D_{\gamma}\left(\tilde{G}^{\alpha \bar{\beta}} \tilde{G}_{\alpha \bar{\beta}}^{\prime}\right)+E_{\gamma}\right)\left(2 D_{\bar{\delta}}\left(\tilde{G}^{\mu \bar{\nu}} \tilde{G}_{\mu \bar{\nu}}^{\prime}\right)+\bar{E}_{\delta}\right) \\
=4 G^{\prime \gamma \bar{\delta}}\left(G^{\alpha \bar{\beta}} D_{\gamma} G_{\alpha \bar{\beta}}^{\prime}\right)\left(G^{\mu \bar{\nu}} D_{\bar{\delta}} G_{\mu \bar{\nu}}^{\prime}\right) \\
\quad+4 G^{\prime \gamma \bar{\delta}}\left(\tilde{G}^{\alpha \bar{\beta}} D_{\gamma} \tilde{G}_{\alpha \bar{\beta}}^{\prime}\right)\left(\tilde{G}^{\mu \bar{\nu}} D_{\bar{\delta}} \tilde{G}_{\mu \bar{\nu}}^{\prime}\right)-2 G^{\prime \gamma \bar{\delta}} E_{\gamma} \bar{E}_{\delta} .
\end{aligned}
$$

From Lemma 3.3,

$$
\begin{aligned}
g^{\prime k l} & \partial_{k}\left(g^{i j} g_{i j}^{\prime}\right) \partial_{l}\left(g^{p q} g_{p q}^{\prime}\right) \\
= & 4 G^{\prime \gamma \bar{\delta}}\left(G^{\alpha \bar{\beta}} D_{\alpha} G_{\gamma \bar{\beta}}^{\prime}\right)\left(G^{\mu \bar{\nu}} D_{\bar{\nu}} G_{\mu \bar{\delta}}^{\prime}\right) \\
& +4 G^{\prime \gamma \bar{\delta}}\left(G^{\alpha \bar{\beta}} D_{\alpha} \tilde{G}_{\gamma \bar{\beta}}^{\prime}\right)\left(G^{\mu \bar{\nu}} D_{\bar{\nu}} \tilde{G}_{\mu \bar{\delta}}^{\prime}\right) \\
& +2 \operatorname{Re}\left\{G^{\prime \gamma \bar{\delta}} G^{\alpha \bar{\beta}}\left(a_{\gamma \alpha \beta}+\sqrt{-1} b_{\gamma \alpha \beta}\right) G^{\mu \bar{\nu}} D_{\bar{\nu}} G_{\mu \bar{\delta}}^{\prime}\right. \\
& \left.+G^{\prime \gamma \bar{\delta}} G^{\alpha \bar{\beta}}\left(a_{\gamma A B}+\sqrt{-1} b_{\gamma A B}\right) G^{\mu \bar{\nu}} D_{\bar{\nu}} \tilde{G}_{\mu \bar{\delta}}^{\prime}\right\} \\
& +\frac{1}{4} G^{\prime \gamma \bar{\delta}} G^{\alpha \bar{\beta}}\left(a_{\gamma \alpha \beta}+\sqrt{-1} b_{\gamma \alpha \beta}\right) G^{\mu \bar{\nu}}\left(a_{\delta \mu \nu}-\sqrt{-1} b_{\delta \mu \nu}\right) \\
& +\frac{1}{4} G^{\prime \gamma \bar{\delta}} G^{\alpha \bar{\beta}}\left(a_{\gamma A B}+\sqrt{-1} b_{\gamma A B}\right) G^{\mu \bar{\nu}}\left(a_{\delta M N}-\sqrt{-1} b_{\delta M N}\right) \\
& -2 G^{\prime \gamma \bar{\delta}} E_{\gamma} \bar{E}_{\delta} .
\end{aligned}
$$

But

$$
\begin{aligned}
& G^{\mu \bar{\nu}} D_{\bar{\nu}} \tilde{G}_{\mu \bar{\delta}}^{\prime} \\
& =G^{\mu \bar{\nu}} D_{\bar{\nu}}^{\prime} G_{\mu \bar{\delta}}^{\prime}+\frac{1}{4} G^{\mu \bar{\nu}}\left(a_{\delta \mu \nu}+a_{\nu M D}-\sqrt{-1} b_{\delta \mu \nu}-\sqrt{-1} b_{\nu M D}\right)-\bar{E}_{\delta},
\end{aligned}
$$


and so, making use of Lemma 3.5,

$$
\begin{aligned}
g^{\prime k l} & \partial_{k}\left(g^{i j} g_{i j}^{\prime}\right) \partial_{l}\left(g^{p q} g_{p q}^{\prime}\right) \\
= & 4 G^{\prime \gamma \bar{\delta}}\left(G^{\alpha \bar{\beta}} D_{\alpha} G_{\gamma \bar{\beta}}^{\prime}\right)\left(G^{\mu \bar{\nu}} D_{\bar{\nu}} G_{\mu \bar{\delta}}^{\prime}\right) \\
& +4 G^{\prime \gamma \bar{\delta}}\left(G^{\alpha \bar{\beta}} D_{\alpha} \tilde{G}_{\gamma \bar{\beta}}^{\prime}\right)\left(G^{\mu \bar{\nu}} D_{\bar{\nu}} \tilde{G}_{\mu \bar{\delta}}^{\prime}\right) \\
& +\frac{1}{2} \operatorname{Re}\left\{G ^ { \prime \gamma \overline { \delta } } G ^ { \alpha \overline { \beta } } ( a _ { \gamma A B } + \sqrt { - 1 } b _ { \gamma A B } ) \left(G ^ { \mu \overline { \nu } } \left(a_{\delta \mu \nu}+a_{\nu M D}\right.\right.\right. \\
& \left.\left.\left.-\sqrt{-1} b_{\delta \mu \nu}-\sqrt{-1} b_{\nu M D}\right)-4 \bar{E}_{\delta}\right)\right\} \\
& +\frac{1}{4} G^{\prime \gamma \bar{\delta}} G^{\alpha \bar{\beta}}\left(a_{\gamma \alpha \beta}+\sqrt{-1} b_{\gamma \alpha \beta}\right) G^{\mu \bar{\nu}}\left(a_{\delta \mu \nu}-\sqrt{-1} b_{\delta \mu \nu}\right) \\
& +\frac{1}{4} G^{\prime \gamma \bar{\delta}} G^{\alpha \bar{\beta}}\left(a_{\gamma A B}+\sqrt{-1} b_{\gamma A B}\right) G^{\mu \bar{\nu}}\left(a_{\delta M N}-\sqrt{-1} b_{\delta M N}\right) \\
& -2 G^{\prime \gamma \bar{\delta}} E_{\gamma} \bar{E}_{\delta} .
\end{aligned}
$$

We can now apply the Cauchy-Schwartz inequality twice to obtain:

$$
\begin{aligned}
& G^{\prime \gamma \bar{\delta}}\left(G^{\alpha \bar{\beta}} D_{\alpha} G_{\gamma \bar{\beta}}^{\prime}\right)\left(G^{\mu \bar{\nu}} D_{\bar{\nu}} G_{\mu \bar{\delta}}^{\prime}\right) \\
& =\sum_{\gamma, \alpha, \mu} G^{\prime \gamma \bar{\gamma}}\left(D_{\alpha} G_{\gamma \bar{\alpha}}^{\prime}\right)\left(D_{\bar{\mu}} G_{\mu \bar{\gamma}}^{\prime}\right) \\
& \leq \sum_{\alpha, \mu}\left(\sum_{\gamma} G^{\prime \gamma \bar{\gamma}}\left|D_{\alpha} G_{\gamma \bar{\alpha}}^{\prime}\right|^{2}\right)^{1 / 2}\left(\sum_{\gamma} G^{\prime \gamma \bar{\gamma}}\left|D_{\mu} G_{\gamma \bar{\mu}}^{\prime}\right|^{2}\right)^{1 / 2} \\
& =\left(\sum_{\alpha}\left(\sum_{\gamma} G^{\prime \gamma \bar{\gamma}}\left|D_{\alpha} G_{\gamma \bar{\alpha}}^{\prime}\right|^{2}\right)^{1 / 2}\right)^{2} \\
& =\left(\sum_{\alpha} \sqrt{G_{\alpha \bar{\alpha}}^{\prime}}\left(\sum_{\gamma} G^{\prime \gamma \bar{\gamma}} G^{\prime \alpha \bar{\alpha}}\left|D_{\alpha} G_{\gamma \bar{\alpha}}^{\prime}\right|^{2}\right)^{1 / 2}\right)^{2} \\
& \leq\left(\sum_{\alpha} G_{\alpha \bar{\alpha}}^{\prime}\right) \sum_{\gamma, \alpha} G^{\prime \gamma \bar{\gamma}} G^{\prime \alpha \bar{\alpha}}\left|D_{\alpha} G_{\gamma \bar{\alpha}}^{\prime}\right|^{2} \\
& \leq 4\left(\operatorname{tr}_{g} g^{\prime}\right) \sum_{\gamma, \alpha, \mu} g^{\prime \gamma \gamma} g^{\prime \alpha \alpha} g^{\mu \mu}\left|D_{\mu} G_{\gamma \bar{\alpha}}^{\prime}\right|^{2} .
\end{aligned}
$$

Similarly,

$$
\begin{aligned}
& G^{\prime \gamma \bar{\delta}}\left(G^{\alpha \bar{\beta}} D_{\alpha} \tilde{G}_{\gamma \bar{\beta}}^{\prime}\right)\left(G^{\mu \bar{\nu}} D_{\bar{\nu}} \tilde{G}_{\mu \bar{\delta}}^{\prime}\right) \\
& \leq 4\left(\operatorname{tr}_{g} g^{\prime}\right) \sum_{\gamma, \alpha, \mu} g^{\prime \gamma \gamma} g^{\prime \alpha \alpha} g^{\mu \mu}\left|D_{\mu} \tilde{G}_{\gamma \bar{\alpha}}^{\prime}\right|^{2} .
\end{aligned}
$$


But

$$
\begin{aligned}
& \sum_{\gamma, \alpha, \mu} g^{\prime \gamma \gamma} g^{\prime \alpha \alpha} g^{\mu \mu}\left|D_{\mu} G_{\gamma \bar{\alpha}}^{\prime}\right|^{2} \\
= & \frac{1}{4} \sum_{\gamma, \alpha, \mu} g^{\prime \gamma \gamma} g^{\prime \alpha \alpha} g^{\mu \mu}\left\{\left(\partial_{\mu} g_{\gamma \alpha}^{\prime}-J_{\mu}{ }^{\prime} g_{j \alpha}^{\prime}\left(\nabla_{i} J_{\gamma}{ }^{j}\right)-J_{\mu}{ }^{i} J_{\gamma}{ }^{j}\left(\nabla_{i} g_{j \alpha}^{\prime}\right)\right)^{2}\right. \\
& \left.+\left(J_{\mu}^{i} \nabla_{i} g_{\gamma \alpha}^{\prime}+g_{j \alpha}^{\prime} \nabla_{\mu} J_{\gamma}{ }^{j}+J_{\gamma}{ }^{j} \nabla_{\mu} g_{j \alpha}^{\prime}\right)^{2}\right\} \\
= & \frac{1}{4} \sum_{\gamma, \alpha, \mu}\left(g^{\prime \gamma \gamma} g^{\prime \alpha \alpha} g^{\mu \mu}\left(\nabla_{\mu} g_{\gamma \alpha}^{\prime}\right)^{2}+g^{\prime \gamma \gamma} g_{\alpha \alpha}^{\prime} g^{M M}\left(\nabla_{M} J_{\gamma}{ }^{\alpha}\right)^{2}\right. \\
& +g^{\prime C C} g^{\prime \alpha \alpha} g^{M M}\left(\nabla_{M} g_{C \alpha}^{\prime}\right)^{2}+g^{\prime \gamma \gamma} g^{\prime \alpha \alpha} g^{M M}\left(\nabla_{M} g_{\gamma \alpha}^{\prime}\right)^{2} \\
& +g^{\prime \gamma \gamma} g_{\alpha \alpha}^{\prime} g^{\mu \mu}\left(\nabla_{\mu} J_{\gamma}{ }^{\alpha}\right)^{2}+g^{\prime C C} g^{\prime \alpha \alpha} g^{\mu \mu}\left(\nabla_{\mu} g_{C \alpha}^{\prime}\right)^{2} \\
& -2 g^{\prime \gamma \gamma} g^{\mu \mu}\left(\nabla_{\mu} g_{\gamma \alpha}^{\prime}\right) J_{\mu}{ }^{i}\left(\nabla_{i} J_{\gamma}{ }^{\alpha}\right)-2 g^{\prime \gamma \gamma} g^{\prime \alpha \alpha} g^{\mu \mu} J_{\mu}{ }^{i} J_{\gamma}{ }^{j}\left(\nabla_{\mu} g_{\gamma \alpha}^{\prime}\right)\left(\nabla_{i} g_{j \alpha}^{\prime}\right) \\
& +2 g^{\prime \gamma \gamma} J_{\gamma}{ }^{\prime} g^{M M}\left(\nabla_{M} J_{\gamma}{ }^{\alpha}\right)\left(\nabla_{M} g_{j \alpha}^{\prime}\right)+2 g^{\prime \gamma \gamma} g^{\mu \mu} J_{\mu}{ }^{\prime}\left(\nabla_{i} g_{\gamma \alpha}^{\prime}\right)\left(\nabla_{\mu} J_{\gamma}{ }^{\alpha}\right) \\
& \left.+2 g^{\prime \gamma \gamma} g^{\prime \alpha \alpha} g^{\mu \mu} J_{\mu}{ }^{i} J_{\gamma}{ }^{j}\left(\nabla_{i} g_{\gamma \alpha}^{\prime}\right)\left(\nabla_{\mu} g_{j \alpha}^{\prime}\right)+2 g^{\prime \gamma \gamma} g^{\mu \mu} J_{\gamma}{ }^{j}\left(\nabla_{\mu} J_{\gamma}{ }^{\alpha}\right)\left(\nabla_{\mu} g_{j \alpha}^{\prime}\right)\right) .
\end{aligned}
$$

A short calculation shows that

$$
\sum_{\gamma, \alpha, \mu} g^{\prime \gamma \gamma} g^{\mu \mu}\left(\nabla_{\mu} g_{\gamma \alpha}^{\prime}\right) J_{\mu}{ }^{i}\left(\nabla_{i} J_{\gamma}{ }^{\alpha}\right)+g^{\prime \gamma \gamma} g^{\prime \alpha \alpha} g^{\mu \mu} J_{\mu}{ }^{i} J_{\gamma}{ }^{j}\left(\nabla_{\mu} g_{\gamma \alpha}^{\prime}\right)\left(\nabla_{i} g_{j \alpha}^{\prime}\right)=0
$$

and similarly that

$$
\sum_{\gamma, \alpha, \mu} g^{\prime \gamma \gamma} g^{\mu \mu} J_{\mu}{ }^{i}\left(\nabla_{i} g_{\gamma \alpha}^{\prime}\right)\left(\nabla_{\mu} J_{\gamma}{ }^{\alpha}\right)+g^{\prime \gamma \gamma} g^{\prime \alpha \alpha} g^{\mu \mu} J_{\mu}{ }^{i} J_{\gamma}{ }^{j}\left(\nabla_{i} g_{\gamma \alpha}^{\prime}\right)\left(\nabla_{\mu} g_{j \alpha}^{\prime}\right)=0 \text {. }
$$

Hence

$$
\begin{aligned}
& 4 \sum_{\gamma, \alpha, \mu} g^{\prime \gamma \gamma} g^{\prime \alpha \alpha} g^{\mu \mu}\left|D_{\mu} G_{\gamma \bar{\alpha}}^{\prime}\right|^{2} \\
& =\frac{1}{4} \sum_{\gamma, \alpha=1}^{2} \sum_{k=1}^{4}\left(g^{\prime \gamma \gamma} g^{\prime \alpha \alpha} g^{k k}\left(\nabla_{k} g_{\gamma \alpha}^{\prime}\right)^{2}+g^{\prime C C} g^{\prime \alpha \alpha} g^{k k}\left(\nabla_{k} g_{C \alpha}^{\prime}\right)^{2}\right. \\
& \left.\quad+2 g^{\prime \gamma \gamma} J_{\gamma}^{j} g^{k k}\left(\nabla_{k} J_{\gamma}^{\alpha}\right)\left(\nabla_{k} g_{j \alpha}^{\prime}\right)+g^{\prime \gamma \gamma} g_{\alpha \alpha}^{\prime} g^{k k}\left(\nabla_{k} J_{\gamma}{ }^{\alpha}\right)^{2}\right) .
\end{aligned}
$$

Similarly, we have

$$
\begin{aligned}
& 4 \sum_{\gamma, \alpha, \mu} g^{\prime \gamma \gamma} g^{\prime \alpha \alpha} g^{\mu \mu}\left|D_{\mu} \tilde{G}_{\gamma \bar{\alpha}}^{\prime}\right|^{2} \\
= & \frac{1}{4} \sum_{\gamma, \alpha=1}^{2} \sum_{k=1}^{4}\left(g^{\prime C C} g^{\prime A A} g^{k k}\left(\nabla_{k} g_{C A}^{\prime}\right)^{2}+g^{\prime \gamma \gamma} g^{\prime A A} g^{k k}\left(\nabla_{k} g_{\gamma A}^{\prime}\right)^{2}\right. \\
& \left.-2 g^{\prime C C} g^{k k}\left(\nabla_{k} J_{C}^{A}\right)\left(\nabla_{k} g_{\gamma A}^{\prime}\right)+g^{\prime C C} g_{A A}^{\prime} g^{k k}\left(\nabla_{k} J_{C}^{A}\right)^{2}\right)
\end{aligned}
$$

Now observe that, at $p$,

$$
\nabla_{k} J_{C}^{A}=-\nabla_{k} J_{\gamma}^{\alpha}
$$


and that

$$
\nabla_{k} g_{\gamma A}^{\prime}+\nabla_{k} g_{C \alpha}^{\prime}=J_{\alpha i}^{\prime}\left(\nabla_{k} J_{C}^{i}\right)+J_{C i}^{\prime}\left(\nabla_{k} J_{\alpha}^{i}\right) .
$$

Using these two simple equalities we obtain at the point $p$, by combining (3.25) and (3.26),

$$
\begin{aligned}
4 & \sum_{\gamma, \alpha, \mu} g^{\prime \gamma \gamma} g^{\prime \alpha \alpha} g^{\mu \mu}\left|D_{\mu} G_{\gamma \bar{\alpha}}^{\prime}\right|^{2}+4 \sum_{\gamma, \alpha, \mu} g^{\prime \gamma \gamma} g^{\prime \alpha \alpha} g^{\mu \mu}\left|D_{\mu} \tilde{G}_{\gamma \bar{\alpha}}^{\prime}\right|^{2} \\
= & \frac{1}{4} g^{\prime k l} g^{\prime i j} g^{p q}\left(\nabla_{p} g_{i k}^{\prime}\right)\left(\nabla_{q} g_{j l}^{\prime}\right)+\frac{1}{4} \sum g^{\prime \gamma \gamma} g_{\alpha \alpha}^{\prime} g^{k k}\left(\nabla_{k} J_{\gamma}^{\alpha}\right)^{2} \\
& +\frac{1}{4} \sum g^{\prime C C} g_{A A}^{\prime} g^{k k}\left(\nabla_{k} J_{C}^{A}\right)^{2} \\
& -\frac{1}{2} \sum g^{\prime C C} g^{k k}\left(\nabla_{k} J_{C}^{A}\right)\left(J_{\alpha i}^{\prime} \nabla_{k} J_{C}^{i}+J_{C i}^{\prime} \nabla_{k} J_{\alpha}^{i}\right) .
\end{aligned}
$$

Using this, together with (3.22), (3.23) and (3.24), we obtain the estimate of Lemma 3.2.

q.e.d.

Returning to the proof of the theorem, we can now combine equations (3.8) with (3.13) and (3.14) together with Lemma 3.2 to obtain

$$
\begin{aligned}
\Delta^{\prime} & \left(\log \operatorname{tr}_{g} g^{\prime}\right) \\
\geq & \frac{1}{\operatorname{tr}_{g} g^{\prime}}\left\{2 \Delta F+2 g^{\prime k l} g^{i j} g^{r s}\left(\nabla_{k} J_{i r}\right)\left(\mathcal{P}_{j s}^{a b} g_{b q}^{\prime} \nabla_{a} J_{l}{ }^{q}-\frac{1}{2} \alpha_{s j q} J_{l}{ }^{q}\right.\right. \\
& \left.-\mathcal{P}_{s j}^{a b} g_{b q}^{\prime} \nabla_{a} J_{l}{ }^{q}\right)+g^{\prime k l} g^{i j} g^{r s}\left(\nabla_{r} J_{i k}\right) J_{a b}^{\prime}\left(J_{l}{ }^{b} \nabla_{s} J_{j}{ }^{a}+J_{j}{ }^{a} \nabla_{s} J_{l}{ }^{b}\right) \\
& -2 g^{\prime k l} g^{i j} g^{r s}\left(\nabla_{i} J_{k r}+\nabla_{k} J_{i r}\right) g_{q l}^{\prime} \nabla_{s} J_{j}{ }^{q} \\
& +2 g^{i j}\left(\nabla_{i} J_{q}{ }^{r}+\nabla_{q} J_{i}{ }^{r}\right)\left(\nabla_{r} J_{j}{ }^{q}\right) \\
& +g^{\prime k l}\left(\nabla_{k} J_{l}{ }^{r}\right)\left(\frac{1}{2} \beta_{i r q} J^{i q}+2 g^{i j} \mathcal{Q}_{i r}^{a b} g_{b q}^{\prime} \nabla_{a} J_{j}{ }^{q}\right. \\
& \left.-g^{i j} g_{i s}^{\prime} \nabla_{j} J_{r}{ }^{s}-g^{i j} J_{r}{ }^{s} J_{p j}^{\prime} \nabla_{s} J_{i}{ }^{p}\right) \\
& +J_{r}{ }^{k} g^{i j} \nabla_{i} \nabla_{j} J_{k}{ }^{r}-2 g^{i j} g^{\prime k l} g_{q j}^{\prime} J_{r}{ }^{q} \nabla_{i} \nabla_{l} J_{k}{ }^{r} \\
& -J_{r}{ }^{q} g_{q j}^{\prime} g^{\prime k l} g^{i j} \nabla_{k} \nabla_{l} J_{i}{ }^{r}-2 R+2 g^{\prime k l} g_{i j}^{\prime} g^{p j} R_{l p k}^{i} \\
& \left.-C^{\prime}\left(\operatorname{tr}_{g} g^{\prime}\right)\left(\operatorname{tr}_{g^{\prime}} g\right)\right\} .
\end{aligned}
$$

From the Calabi-Yau equation and the arithmetic-geometric means inequality we have

$$
\operatorname{tr}_{g} g^{\prime} \geq 4 \exp \left(\frac{\inf _{M} F}{2}\right)>0 .
$$

Hence, recalling from section 2 that $\|J\|_{C^{2}}$ can be bounded in terms of $\|\operatorname{Rm}(g)\|_{C^{2}}$, we have:

$$
\Delta^{\prime}\left(\log \operatorname{tr}_{g} g^{\prime}\right) \geq-\frac{A}{2} \operatorname{tr}_{g^{\prime}} g-A,
$$

for a constant $A$ depending only on $\|\operatorname{Rm}(g)\|_{C^{2}}$, sup $|F|$ and the lower bound of $\Delta F$. We now apply the maximum principle to the quantity 
$\left(\log \operatorname{tr}_{g} g^{\prime}-A \phi_{1}\right)$. Suppose that the maximum is achieved at a point $x_{0}$ on $M$. Then at $x_{0}$ we have

$$
\Delta^{\prime}\left(\log \operatorname{tr}_{g} g^{\prime}-A \phi_{1}\right) \leq 0 .
$$

Recall that $\Delta^{\prime} \phi_{1}=4-\operatorname{tr}_{g^{\prime}} g$. At $x_{0}$ we see that from (3.27)

$$
\begin{aligned}
0 & \geq \Delta^{\prime}\left(\log \operatorname{tr}_{g} g^{\prime}-A \phi_{1}\right) \\
& \geq \frac{A}{2} \operatorname{tr}_{g^{\prime}} g-5 A,
\end{aligned}
$$

so that $\left(\operatorname{tr}_{g^{\prime}} g\right)\left(x_{0}\right) \leq 10$. On the other hand, from (2.8), we have that

$$
\left(\operatorname{tr}_{g} g^{\prime}\right)\left(x_{0}\right) \leq 10 e^{F\left(x_{0}\right)}
$$

and thus at any point $x$ we have

$$
\log \left(\left(\operatorname{tr}_{g} g^{\prime}\right)(x)\right)-A \phi_{1}(x) \leq \log 10 e^{F\left(x_{0}\right)}-A \phi_{1}\left(x_{0}\right) .
$$

The theorem follows after exponentiating.

q.e.d.

\section{Hölder estimate on the metric}

In this section we will prove a Hölder estimate on $g^{\prime}$ given a uniform estimate of $g^{\prime}$, using a modification of the method of Evans $[\mathbf{E v}]$ and Krylov $[\mathbf{K r}]$ (see also the estimate of Trudinger $[\mathbf{T r} \mathbf{2}]$ and the exposition of Siu $[\mathbf{S i}])$.

Theorem 4.1. Suppose that $g^{\prime}$ satisfies the equation (1.2) and there exists a constant $C_{0}$ with

$$
C_{0}^{-1} g \leq g^{\prime} \leq C_{0} g
$$

Then there exist positive constants $C$ and $\alpha$ depending only on $g, C_{0}$ and $\|F\|_{C^{2}(g)}$ such that

$$
\left\|\operatorname{tr}_{g} g^{\prime}\right\|_{C^{\alpha}(g)} \leq C
$$

Proof. We will work locally and fix a coordinate system $\left(x^{1}, \cdots, x^{4}\right)$ with the same properties as the one in the proof of Lemma 3.2, with the point $p$ corresponding to $x=0$. We will show that, with the notation of section 3 ,

$$
\left|G^{\alpha \bar{\beta}} G_{\alpha \bar{\beta}}^{\prime}(y)-G^{\alpha \bar{\beta}} G_{\alpha \bar{\beta}}^{\prime}(x)\right| \leq C^{\prime} R^{\alpha},
$$

for all $x, y \in B_{R}(0)$ and $0<R<R_{0} / 2$ for some positive constants $\alpha$, $R_{0}$ and $C^{\prime}$, where $B_{R}(0)$ is the ball of radius $R$ centred at 0 . This will prove the theorem, since a short calculation shows

$$
G^{\alpha \bar{\beta}} G_{\alpha \bar{\beta}}^{\prime}=\frac{1}{2} g^{i j} g_{i j}^{\prime}+O(R) .
$$

To prove (4.1), first note that, by a straightforward calculation,

$$
\left(\operatorname{det} G^{\prime}\right)^{2}=\frac{1}{16} \operatorname{det} g^{\prime}+\eta
$$


where $\eta=\eta(x)$ is a function of the form

$$
\eta=\sum_{a, b, c, d=1}^{4} h_{a b c d} G_{a}^{\prime} G_{b}^{\prime} G_{c}^{\prime} G_{d}^{\prime},
$$

where $G_{1}^{\prime}=G_{1 \overline{1}}^{\prime}, G_{2}^{\prime}=G_{2 \overline{2}}^{\prime}, G_{3}^{\prime}=G_{1 \overline{2}}^{\prime}+G_{2 \overline{1}}^{\prime}$ and $G_{4}^{\prime}=\sqrt{-1}\left(G_{1 \overline{2}}^{\prime}-G_{2 \overline{1}}^{\prime}\right)$, and where the $h_{a b c d}$ are smooth functions depending only on $J$ which vanish at 0 . Note that here, and in the sequel, we are shrinking $R_{0}$ whenever necessary. Writing

$$
K=\frac{1}{16} e^{2 F} \operatorname{det} g,
$$

and using the equation (3.1), we see that

$$
2 \log \operatorname{det} G^{\prime}=\log (K+\eta) .
$$

Define a function $\Phi$ on the space of positive definite Hermitian matrices by $\Phi\left(A_{\alpha \bar{\beta}}\right)=2 \log \operatorname{det}\left(A_{\alpha \bar{\beta}}\right)$. Since $\Phi$ is concave, the tangent plane to the graph of $\Phi$ at a point $G_{\alpha \bar{\beta}}^{\prime}(y)$ lies above the graph of $\Phi$ and so

$$
2 G^{\prime \alpha \bar{\beta}}(y)\left(G_{\alpha \bar{\beta}}^{\prime}(y)-G_{\alpha \bar{\beta}}^{\prime}(x)\right) \leq \Phi\left(G_{\alpha \bar{\beta}}^{\prime}(y)\right)-\Phi\left(G_{\alpha \bar{\beta}}^{\prime}(x)\right),
$$

for $x, y$ in $\bar{B}_{2 R}(0)$. From (4.2),

$$
\begin{aligned}
2 G^{\prime \alpha \bar{\beta}}(y)\left(G_{\alpha \bar{\beta}}^{\prime}(y)-G_{\alpha \bar{\beta}}^{\prime}(x)\right) & \leq \log \left(1+\frac{K(y)-K(x)+\eta(y)-\eta(x)}{K(x)+\eta(x)}\right) \\
& \leq \log \left(1+C_{1} R\right) \\
& \leq C_{1} R .
\end{aligned}
$$

We now need the following elementary linear algebra lemma.

Lemma 4.1. Let $S(\lambda, \Lambda)$ be the set of $2 \times 2$ positive definite Hermitian matrices with eigenvalues between $\lambda$ and $\Lambda$, with $0<\lambda \leq \Lambda$. Then there exist a finite number of bases of unit vectors $\left\{\left(V_{\nu}^{(1)}, V_{\nu}^{(2)}\right)\right\}_{\nu=1}^{N}$ and constants $0<\lambda^{*}<\Lambda^{*}$ depending only on $\lambda$ and $\Lambda$ such that any $A$ in $S(\lambda, \Lambda)$ can be written

$$
A=\sum_{\nu=1}^{N} \beta_{\nu}\left(V_{\nu}^{(1)} \otimes \bar{V}_{\nu}^{(1)}+V_{\nu}^{(2)} \otimes \bar{V}_{\nu}^{(2)}\right)
$$

with $\lambda^{*} \leq \beta_{\nu} \leq \Lambda^{*}$.

Proof. This lemma can be proved by a straightforward modification of the argument in $[\mathbf{M o W a}]$.

q.e.d.

Using this lemma we see that

$$
G^{\alpha \bar{\beta}}(y)=\sum_{\nu=1}^{N} \beta_{\nu}(y)\left(V_{\nu}^{(1)} \otimes \bar{V}_{\nu}^{(1)}+V_{\nu}^{(2)} \otimes \bar{V}_{\nu}^{(2)}\right),
$$


for $\lambda^{*}<\beta_{\nu}<\Lambda^{*}$ where the $V_{\nu}^{(i)}$ and $\lambda^{*}$ and $\Lambda^{*}$ depend only on the constant $C_{0}$. Define

$$
w_{\nu}=\left(\left(V_{\nu}^{(1)}\right)^{\alpha} \overline{\left(V_{\nu}^{(1)}\right)^{\beta}}+\left(V_{\nu}^{(2)}\right)^{\alpha} \overline{\left(V_{\nu}^{(2)}\right)^{\beta}}\right) G_{\alpha \bar{\beta}}^{\prime},
$$

where $\left(V_{\nu}^{(i)}\right)^{\alpha}$ is the $\alpha$-component of the vector $V_{\nu}^{(i)}$. We can then rewrite (4.3) as

$$
\sum_{\nu=1}^{N} \beta_{\nu}(y)\left(w_{\nu}(y)-w_{\nu}(x)\right) \leq C_{1} R, \quad \text { for } x, y \in \bar{B}_{2 R}(0) .
$$

We will now use the concavity of $\Phi$ again, this time to derive a differential inequality for $w_{\nu}$. For each $\nu$, apply the operator $\sum_{i=1}^{2} D_{\nu}^{(i)} D_{\bar{\nu}}^{(i)}=$ $\sum_{i=1}^{2}\left(V_{\nu}^{(i)}\right)^{\gamma} \overline{\left(V_{\nu}^{(i)}\right)^{\delta}} D_{\gamma} D_{\bar{\delta}}$ to (4.2) to obtain:

$$
\begin{aligned}
& \sum_{i=1}^{2}\left(2 G^{\prime \alpha \bar{\beta}} D_{\nu}^{(i)} D_{\bar{\nu}}^{(i)} G_{\alpha \bar{\beta}}^{\prime}-2 G^{\prime \alpha \bar{\gamma}} G^{\prime \sigma \bar{\beta}}\left(D_{\nu}^{(i)} G_{\sigma \bar{\gamma}}^{\prime}\right)\left(D_{\bar{\nu}}^{(i)} G_{\alpha \bar{\beta}}^{\prime}\right)\right) \\
& =\sum_{i=1}^{2}\left(\frac{D_{\nu}^{(i)} D_{\bar{\nu}}^{(i)}(K+\eta)}{K+\eta}-\frac{\left|D_{\nu}^{(i)}(K+\eta)\right|^{2}}{(K+\eta)^{2}}\right) .
\end{aligned}
$$

Apply Lemma 3.3 twice to the first term on the left hand side and the first term on the right hand side of (4.5). Making use of the good second term on the left hand side of (4.5) we see that there is a second order elliptic operator $L_{\nu}=a^{i j} \partial_{i} \partial_{j}$ with $C_{2}^{-1}|\xi|^{2} \leq a^{i j} \xi_{i} \xi_{j} \leq C_{2}|\xi|^{2}$ such that

$$
L_{\nu} w_{\nu} \geq-C_{3} .
$$

From the inequalities (4.4) and (4.6) we make the following claim.

Claim. There exist positive constants $\hat{C}$ and $\delta$ such that

$$
\operatorname{Osc}_{B_{R}(0)} w_{\nu} \leq \hat{C} R^{\delta}, \quad \text { for } 0<R<R_{0} / 2 .
$$

Of course, given this claim, we are finished, since we can then write

$$
G^{\alpha \bar{\beta}} G_{\alpha \bar{\beta}}^{\prime}=\sum_{\nu=1}^{N} \hat{\beta}_{\nu} w_{\nu}
$$

with $\hat{\beta}_{\nu}$ smooth bounded functions depending only on $g$ and $J$ and satisfying $C_{4}^{-1}<\hat{\beta}_{\nu}<C_{4}$. This gives (4.1) and Theorem 4.1 follows.

Proof of Claim. Although this proof can easily be extracted from [Tr2] (see also [Si]), we will include a sketch of the argument here for the convenience of the reader. The key tool is the following Harnack inequality 
[Tr1]: if $u \geq 0$ satisfies $L u=a^{i j} \partial_{i} \partial_{j} u \leq C_{3}$ with $C_{2}^{-1}|\xi|^{2} \leq a^{i j} \xi_{i} \xi_{j} \leq$ $C_{2}|\xi|^{2}$ on $B_{2 R}(0)$ then there exists $p>0$ such that

$$
\left(\frac{1}{R^{4}} \int_{B_{R}(0)} u^{p}\right)^{1 / p} \leq C_{H}\left(\inf _{B_{R}(0)} u+R\right),
$$

where the constant $C_{H}$ depends only on $C_{2}$ and $C_{3}$.

Set $M_{s \nu}=\sup _{B_{s R}(0)} w_{\nu}$ and $m_{s \nu}=\inf _{B_{s R}(0)} w_{\nu}$ for $s=1,2$ and apply the Harnack inequality to $\left(M_{2 \nu}-w_{\nu}\right)$ to obtain, for fixed $l$,

$$
\begin{aligned}
& \left(\frac{1}{R^{4}} \int_{B_{R}(0)}\left(\sum_{\nu \neq l}\left(M_{2 \nu}-w_{\nu}\right)\right)^{p}\right)^{1 / p} \\
& \leq N^{1 / p} \sum_{\nu \neq l}\left(\frac{1}{R^{4}} \int_{B_{R}(0)}\left(M_{2 \nu}-w_{\nu}\right)^{p}\right)^{1 / p} \\
& \leq C_{5}\left(\sum_{\nu \neq l}\left(M_{2 \nu}-M_{1 \nu}\right)+R\right) \\
& \leq C_{5}(\omega(2 R)-\omega(R)+R),
\end{aligned}
$$

where $\omega(s R)=\sum_{\nu=1}^{N} \operatorname{Osc}_{B_{s R}(0)} w_{\nu}=\sum_{\nu=1}^{N}\left(M_{s \nu}-m_{s \nu}\right)$. From (4.4) we have

$$
\beta_{l}\left(w_{l}(y)-w_{l}(x)\right) \leq C_{1} R+\sum_{\nu \neq l} \beta_{\nu}\left(w_{\nu}(x)-w_{\nu}(y)\right),
$$

for $x, y \in \bar{B}_{2 R}(0)$. Choosing $x \rightarrow m_{2 l}$ and integrating over $y \in B_{R}(0)$ gives

$$
\begin{aligned}
& \left(\frac{1}{R^{4}} \int_{B_{R}(0)}\left(w_{l}-m_{2 l}\right)^{p}\right)^{1 / p} \\
& \leq C_{6} R+C_{6}\left(\frac{1}{R^{4}} \int_{B_{R}(0)}\left(\sum_{\nu \neq l}\left(M_{2 \nu}-w_{\nu}\right)^{p}\right)\right)^{1 / p} \\
& \leq C_{7}(\omega(2 R)-\omega(R)+R) .
\end{aligned}
$$

Now apply the Harnack inequality to $\left(M_{2 l}-w_{l}\right)$ to obtain

$$
\begin{aligned}
\left(\frac{1}{R^{4}} \int_{B_{R}(0)}\left(M_{2 l}-w_{l}\right)^{p}\right)^{1 / p} & \leq C_{8}\left(M_{2 l}-M_{1 l}+R\right) \\
& \leq C_{8}(\omega(2 R)-\omega(R)+R) .
\end{aligned}
$$

Combining (4.8) and (4.9) we see that

$$
M_{2 l}-m_{2 l} \leq C_{9}(\omega(2 R)-\omega(R)+R),
$$


and summing in $l$ gives

$$
\omega(2 R) \leq C_{10}(\omega(2 R)-\omega(R)+R),
$$

from which it follows that

$$
\omega(R) \leq\left(\frac{C_{10}-1}{C_{10}}\right) \omega(2 R)+R,
$$

and the claim follows by a well-known argument (see [GiTr], Chapter $8)$.

q.e.d.

\section{Higher order estimates}

In this section we will prove estimates on $g^{\prime}$ and all of its derivatives given a Hölder estimate

$$
\left\|\operatorname{tr}_{g} g^{\prime}\right\|_{C^{\alpha}(g)} \leq C
$$

with $0<\alpha<1$ and an estimate $g^{\prime} \geq C^{-1} g$. In light of Theorem 3.1 and Theorem 4.1 this will complete the proof of Theorem 1.

Consider the normalized almost-Kähler potential $\phi_{0}$ defined by (see Section 2)

$$
\Delta \phi_{0}=\operatorname{tr}_{g} g^{\prime}-4, \quad \int_{M} \phi_{0} \frac{\omega^{2}}{2}=0 .
$$

From (5.1), by the elliptic Schauder estimates for the Laplacian we have

$$
\left\|\phi_{0}\right\|_{C^{2+\alpha}(g)} \leq C_{0}
$$

Recall that the 1-form $a_{0}$ satisfies

$$
\omega^{\prime}=\omega-\frac{1}{2} d\left(J d \phi_{0}\right)+d a_{0} .
$$

Without loss of generality, we can assume that $a_{0}$ is $L^{2}$ orthogonal to the harmonic 1-forms. Then since $a_{0}$ satisfies the uniformly elliptic system (2.7) for $s=0$ and is orthogonal to its kernel we can use (5.2) and the Schauder elliptic estimates to obtain

$$
\left\|a_{0}\right\|_{C^{2+\alpha}(g)} \leq C_{1} .
$$

It follows that $\left\|g^{\prime}\right\|_{C^{\alpha}(g)} \leq C_{2}$. Differentiating the Calabi-Yau equation (3.1), we see that

$$
g^{\prime i j} \partial_{i} \partial_{j}\left(\partial_{k} \phi_{0}\right)+\{\text { lower order terms }\}=g^{i j} \partial_{k} g_{i j}+2 \partial_{k} F,
$$

where the lower order terms may contain up to two derivatives of $\phi_{0}$ or $a_{0}$. Since the coefficients of this elliptic equation are in $C^{\alpha}$ we can apply the standard Schauder estimates again to obtain

$$
\left\|\phi_{0}\right\|_{C^{3+\alpha}(g)} \leq C_{3} .
$$

From (2.7) we then obtain

$$
\left\|a_{0}\right\|_{C^{3+\alpha}(g)} \leq C_{4}
$$


The rest of the higher order estimates follow from (5.3), (2.7) and a bootstrapping argument. This completes the proof of Theorem 1 .

\section{Proof of Theorem 2: the case $b^{+}(M)=1$}

For this section we assume $b^{+}(M)=1$. Consider the equation

$$
\omega_{t}^{\prime 2}=e^{t F+c_{t}} \omega^{2} \text {, }
$$

where $c_{t}$ is the constant given by $c_{t}=\log \left(\int_{M} \omega^{2} / \int_{M} e^{t F} \omega^{2}\right)$, and where $\omega_{t}^{\prime}$ is required to be cohomologous to $\omega$ and compatible with $J$. Let

$$
T=\left\{t^{\prime} \in[0,1] \mid \exists \text { smooth } \omega_{t}^{\prime} \text { solving (6.1) for } t \in\left[0, t^{\prime}\right]\right\} .
$$

Clearly $0 \in T$. We will show that $T$ is both open and closed in $[0,1]$. This will prove Theorem 2 . Note that if $\omega_{t}^{\prime}$ is in $C^{\alpha}$ then by the estimates of section 5 it is smooth.

We show now that $T$ is open. Fix $t_{0}$ in $T$. We will show that (6.1) can be solved for $t$ in an open neighbourhood containing $t_{0}$. Fix $\tilde{\omega}=\omega_{t_{0}}^{\prime}$. Then solving (6.1) near $t_{0}$ is equivalent to solving

$$
\log \frac{\omega_{t}^{\prime 2}}{\tilde{\omega}^{2}}-\left(t-t_{0}\right) F-\left(c_{t}-c_{t_{0}}\right)=0
$$

for $t$ close to $t_{0}$.

Let $\Lambda^{k, s+\alpha}$ be the space of $k$-forms in $C^{s+\alpha}$, and let $W^{\alpha} \subset \Lambda_{\tilde{\omega}}^{+}$be the space of self-dual two forms $\gamma$ in $C^{\alpha}$ satisfying $\int_{M} \exp \left(\frac{2 \gamma \wedge \tilde{\omega}}{\tilde{\omega}^{2}}\right) \tilde{\omega}^{2}=$ $\int_{M} \tilde{\omega}^{2}$. Then define a map

$$
\Phi: \Lambda^{1,1+\alpha} \times \mathbb{R} \rightarrow W^{\alpha},
$$

by

$$
\Phi(b, t)=\left(\log \frac{(\tilde{\omega}+d b)^{2}}{\tilde{\omega}^{2}}-\left(t-t_{0}\right) F-\hat{c}\right) \frac{\tilde{\omega}}{2}+\mathcal{P} d b
$$

where

$$
\hat{c}(b, t)=\log \left(\int_{M} e^{-\left(t-t_{0}\right) F}(\tilde{\omega}+d b)^{2}\right)-\log \int_{M} \omega^{2} .
$$

Note that if we can find $b=b(t)$ solving $\Phi(b, t)=0$ for $t$ close to $t_{0}$, then this would imply $\hat{c}=c_{t}-c_{t_{0}}$ and complete the openness argument.

Since $b^{+}(M)=1$, the space $\mathcal{H}_{\tilde{\omega}}^{+}$of harmonic self-dual 2-forms with respect to $\tilde{\omega}$ is spanned by $\tilde{\omega}$. Notice that the tangent space to $W^{\alpha}$ at $\Phi\left(0, t_{0}\right)$ is equal to $\left(\mathcal{H}_{\tilde{\omega}}^{+}\right)^{\perp} \cap \Lambda^{2, \alpha}$, where $\left(\mathcal{H}_{\tilde{\omega}}^{+}\right)^{\perp} \subset \Lambda_{\tilde{\omega}}^{+}$is the space of selfdual 2 -forms which are $L^{2}(\tilde{\omega})$ orthogonal to $\mathcal{H}_{\tilde{\omega}}^{+}$. Then the derivative of $\Phi$ in the $b$-variable at $\left(0, t_{0}\right)$ is a map

$$
\left(D_{1} \Phi\right)_{\left(0, t_{0}\right)}: \Lambda^{1,1+\alpha} \rightarrow\left(\mathcal{H}_{\tilde{\omega}}^{+}\right)^{\perp} \cap \Lambda^{2, \alpha}
$$

given by

$$
\left(D_{1} \Phi\right)_{\left(0, t_{0}\right)}(\beta)=d_{\tilde{\omega}}^{+} \beta
$$


It is well known (see [DoKr], for example) that this map is surjective and hence openness follows by the implicit function theorem.

We now need to prove closedness under the assumption that the Nijenhuis tensor is small in the $L^{1}$ sense. Note that from the discussion in section 2 , since $|\nabla N(J)|$ is uniformly bounded in terms of the curvature of $g$, if the Nijenhuis tensor is small in the $L^{1}$ norm, it is small in the $C^{0}$ norm, and hence also in the $L^{p}$ norm for any $p>1$. It will be convenient (and sufficient) for us to prove Theorem 2 under the assumption that $N(J)$ is small in some $L^{p}$ norm, where $p$ will be a fixed constant strictly larger than 2.

We will use the following lemma.

Lemma 6.1. Let $\omega^{\prime}=\omega-\frac{1}{2} d\left(J d \phi_{1}\right)+d a_{1}$ be a solution of the CalabiYau equation (1.2). Suppose that for some constants $p>2$ and $B$,

$$
\left(\int_{M}\left|\frac{d a_{1} \wedge \omega}{\omega^{2}}\right|^{p} \omega^{2}\right)^{1 / p} \leq B .
$$

Then there exists a constant $C^{\prime}$ depending only on $g, p, B$ and $\sup _{M}|F|$ such that

$$
\sup _{M} \phi_{1}-\inf _{M} \phi_{1} \leq C^{\prime} .
$$

Proof. This is a modification of Yau's well-known Moser iteration argument. For ease of notation, write $\phi=\phi_{1}$. Assume that $\int_{M} \phi \omega^{2}=0$. For $l \geq 0$,

$$
\begin{aligned}
C_{0} \int_{M}|\phi|^{l+1} \omega^{2} \geq & \int_{M} \phi|\phi|^{l}\left(\omega^{2}-\omega^{\prime 2}\right) \\
= & \frac{1}{2} \int_{M} \phi|\phi|^{l} d(J d \phi) \wedge\left(\omega+\omega^{\prime}\right)-\int_{M} \phi|\phi|^{l} d a_{1} \wedge \omega \\
= & -\frac{(l+1)}{2} \int_{M}|\phi|^{l} d \phi \wedge J d \phi \wedge\left(\omega+\omega^{\prime}\right) \\
& -\int_{M} \phi|\phi|^{l} d a_{1} \wedge \omega \\
= & -\frac{(l+1)}{2(l / 2+1)^{2}} \int_{M} d\left(\phi|\phi|^{l / 2}\right) \wedge J d\left(\phi|\phi|^{l / 2}\right) \wedge\left(\omega+\omega^{\prime}\right) \\
& -\int_{M} \phi|\phi|^{l} d a_{1} \wedge \omega \\
\geq & \frac{(l+1)}{4(l / 2+1)^{2}} \int_{M}\left|\nabla\left(\phi|\phi|^{l / 2}\right)\right|^{2} \omega^{2} \\
& -\left(\int_{M}|\phi|^{q(l+1)} \omega^{2}\right)^{1 / q}\left(\int_{M}\left|\frac{d a_{1} \wedge \omega}{\omega^{2}}\right|^{p} \omega^{2}\right)^{1 / p},
\end{aligned}
$$


for $q$ satisfying $1 / p+1 / q=1$. Setting $l=0$ we see that since $q<2$,

$$
\int_{M}|\nabla \phi|^{2} \leq C_{1}\left(\left(\int_{M}|\phi|^{q}\right)^{1 / q}+\int_{M}|\phi|\right) \leq C_{2}\left(\left(\int_{M}|\phi|^{2}\right)^{1 / 2}+1\right),
$$

where we have omitted the volume form $\omega^{2}$. Since $\int_{M} \phi=0$ we obtain $\|\phi\|_{L^{2}} \leq C_{3}$ from the Poincaré inequality.

We have for general $l$,

$$
\begin{aligned}
& \int_{M}\left|\nabla\left(\phi|\phi|^{l / 2}\right)\right|^{2} \\
& \leq C_{4}(l+2) \max \left\{1, \int_{M}|\phi|^{l+2},\left(\int_{M}|\phi|^{q(l+1)}\right)^{1 / q}\right\} .
\end{aligned}
$$

The Sobolev inequality gives

$$
\left(\int_{M}|u|^{4}\right)^{1 / 2} \leq C_{5}\left(\int_{M}|\nabla u|^{2}+\int_{M} u^{2}\right),
$$

for functions $u$ on $M$. Set $r=l+2 \geq 2$. Applying the Sobolev inequality to $u=\phi|\phi|^{l / 2}$, making use of (6.3) and raising to the power $1 / r$ gives

$$
\|\phi\|_{L^{2 r}} \leq C_{6}^{1 / r} r^{1 / r} \max \left\{1,\|\phi\|_{L^{r}},\|\phi\|_{L^{q(r-1)}}^{(r-1) / r}\right\} .
$$

Setting $r=2$ we obtain $\|\phi\|_{L^{4}} \leq C_{7}$. For general $r$ we use that fact that $\|\phi\|_{L^{a}} \leq C_{8}\|\phi\|_{L^{b}}$ whenever $a \leq b$ to see that

$$
\|\phi\|_{L^{2 r}} \leq C_{9}^{1 / r} r^{1 / r} \max \left\{1,\|\phi\|_{L^{q r}}\right\} .
$$

By successively replacing $r$ by $\sigma r$ for $\sigma=2 / q>1$ we see that for all $k=0,1,2, \ldots$,

$$
\|\phi\|_{L^{2 r \sigma^{k}}} \leq C_{9}^{\left(\frac{1}{r} \sum_{i=0}^{k} \sigma^{-i}\right)} r^{\left(\frac{1}{r} \sum_{i=0}^{k} \sigma^{-i}\right)} \sigma^{\left(\frac{1}{r} \sum_{i=1}^{k} i \sigma^{-i}\right)} \max \left\{1,\|\phi\|_{L^{q r}}\right\} .
$$

Set $r=2$ and let $k \rightarrow \infty$. This gives a bound

$$
\|\phi\|_{C^{0}} \leq C_{10} \max \left\{1,\|\phi\|_{L^{2 q}}\right\},
$$

which is uniformly bounded since $q<2$. This completes the proof of the lemma.

q.e.d.

Remark 6.1. In a private discussion, Donaldson made the following surprising observation: the almost-Kähler potential $\phi_{1 / 2}$ is uniformly bounded, without any assumption on $N(J)$. This can be proved using a Moser iteration argument and equation (2.5).

It is now not difficult to complete the proof of Theorem 2. We suppose that we have a solution of $(6.1)$ for $t \in\left[0, t_{0}\right)$ for some $t_{0} \in[0,1)$. We require uniform estimates on $\omega_{t}^{\prime}$ and all its derivatives and by Theorem 1 , it is sufficient to obtain a uniform estimate of $\phi_{1}$. We have the following claim. 
Claim. Let $p>2$. There exists $\epsilon>0$ depending only on $p, g$ and $\|F\|_{C^{2}(g)}$ such that if $\|N(J)\|_{L^{p}}<\epsilon$ then for $t \in\left[0, t_{0}\right), a_{1}=a_{1}(t)$ satisfies

$$
\left(\int_{M}\left|\frac{d a_{1} \wedge \omega}{\omega^{2}}\right|^{p} \omega^{2}\right)^{1 / p}<1
$$

Proof of Claim. At $t=0$ we have $d a_{1}=0$. Suppose that the claim is false. Then there is a first time $t^{\prime} \in\left[0, t_{0}\right)$ with

$$
\left(\int_{M}\left|\frac{d a_{1} \wedge \omega}{\omega^{2}}\right|^{p} \omega^{2}\right)^{1 / p}=1
$$

Then it follows from Lemma 6.1 that we have a Hölder estimate on $\omega^{\prime}$ at $t=t^{\prime}$. Now the $L^{p}$ a priori estimates for the elliptic system (2.7) with $s=1$ give

$$
\left(\int_{M}\left|\frac{d a_{1} \wedge \omega}{\omega^{2}}\right|^{p} \omega^{2}\right)^{1 / p} \leq K\|N(J)\|_{L^{p}},
$$

for some uniform constant $K$. Picking $\epsilon=1 / 2 K$ gives a contradiction and proves the claim.

q.e.d.

Then the first part of Theorem 2 follows from this claim and the previous lemma.

\section{Proof of Theorem 2: the case $b^{+}(M)>1$}

Suppose that $b^{+}(M)=r+1$. We begin with the openness argument. For convenience, assume that $\omega$ has been scaled so that $\int_{M} \omega^{2}=1$. We wish to solve the equation

$$
\omega_{t}^{\prime 2}=e^{t F+c_{t}} \omega^{2},
$$

with $c_{t}=-\log \left(\int_{M} e^{t F} \omega^{2}\right)$, for $\omega_{t}^{\prime}$ satisfying $\int_{M} \omega_{t}^{\prime} \wedge \omega>0$ and $\left[\omega_{t}^{\prime}\right] \in H_{\omega}^{+}$. As in section 6 , we suppose that there is a solution $\tilde{\omega}=\omega_{t_{0}}^{\prime}$ at $t=t_{0}$ and show that (7.1) can be solved for $t$ close to $t_{0}$.

Let $\chi_{1}, \ldots, \chi_{r}$ and $\tilde{\chi}_{1}, \ldots, \tilde{\chi}_{r}$ be self-dual harmonic 2 -forms with respect to $\omega$ and $\tilde{\omega}$ respectively such that $\left\{\omega, \chi_{1}, \ldots, \chi_{r}\right\}$ and $\left\{\tilde{\omega}, \tilde{\chi}_{1}, \ldots\right.$, $\left.\tilde{\chi}_{r}\right\}$ are $L^{2}$ orthonormal bases for $\mathcal{H}_{\omega}^{+}$and $\mathcal{H}_{\tilde{\omega}}^{+}$. Let $\Lambda^{1,1+\alpha}$ and $W^{\alpha}$ be as in section 6. Consider the operator $\Phi: \Lambda^{1,1+\alpha} \times \mathbb{R}^{r} \times \mathbb{R} \rightarrow W^{\alpha}$ given by

$$
\begin{aligned}
\Phi(b, \underline{s}, t)= & \left(\log \frac{\left(\tilde{\omega}+\sum_{i=1}^{r} s_{i} \chi_{i}+d b\right)^{2}}{\tilde{\omega}^{2}}-\left(t-t_{0}\right) F-\hat{c}\right) \frac{\tilde{\omega}}{2} \\
& +\mathcal{P}\left(\sum_{i=1}^{r} s_{i} \chi_{i}+d b\right),
\end{aligned}
$$


where

$$
\hat{c}(b, \underline{s}, t)=\log \left(\int_{M} e^{-\left(t-t_{0}\right) F}\left(\tilde{\omega}+\sum_{i=1}^{r} s_{i} \chi_{i}+d b\right)^{2}\right) .
$$

We have a solution $\Phi\left(0, \underline{0}, t_{0}\right)=0$ and if we can find $b$ and $\underline{s}$ depending on $t$ solving $\Phi(b, \underline{s}, t)=0$ for $t$ near $t_{0}$, then after rescaling we would have our desired solution. Write $\Pi_{\left\langle\tilde{\chi}_{1}, \ldots, \tilde{\chi}_{r}\right\rangle}$ for the $L^{2}(\tilde{\omega})$ projection onto the space spanned by $\tilde{\chi}_{1}, \ldots, \tilde{\chi}_{r}$. Define $\Psi_{1}: \Lambda^{1,1+\alpha} \times \mathbb{R}^{r} \times \mathbb{R} \rightarrow$ $W^{\alpha} \cap\left(\left\langle\tilde{\chi}_{1}, \ldots, \tilde{\chi}_{r}\right\rangle\right)^{\perp}$ by

$$
\Psi_{1}(b, \underline{s}, t)=\left(1-\Pi_{\left\langle\tilde{\chi}_{1}, \ldots, \tilde{\chi}_{r}\right\rangle}\right) \Phi(b, \underline{s}, t) .
$$

The derivative $\left(D_{1} \Psi_{1}\right)_{\left(0, \underline{\underline{0}} t_{0}\right)}: \Lambda^{1,1+\alpha} \rightarrow\left(\mathcal{H}_{\tilde{\omega}}^{+}\right)^{\perp} \cap \Lambda^{2, \alpha}$ is given by

$$
\left(D_{1} \Psi_{1}\right)_{\left(0, \underline{0}, t_{0}\right)}(\beta)=d_{\tilde{\omega}}^{+} \beta .
$$

This map is surjective and so by the implicit function theorem, given $(\underline{s}, t)$ near $\left(\underline{0}, t_{0}\right) \in \mathbb{R}^{r} \times \mathbb{R}$ there exists $b=b(\underline{s}, t)$ solving $\Psi_{1}=0$. Now define a map $\Psi_{2}: \mathbb{R}^{r} \times \mathbb{R} \rightarrow \mathbb{R}^{r}$ in a neighbourhood of $\left(\underline{0}, t_{0}\right)$ by

$$
\Psi_{2}(\underline{s}, t)=\Pi_{\left\langle\tilde{\chi}_{1}, \ldots, \tilde{\chi}_{r}\right\rangle} \Phi(b(\underline{s}, t), \underline{s}, t),
$$

where we are identifying $\mathbb{R}^{r}$ and the space spanned by the $\tilde{\chi}_{i}$. Calculate

$$
\begin{aligned}
\left(\left(D_{1} \Psi_{2}\right)_{\left(\underline{0}, t_{0}\right)}\right)_{i j} & =\int_{M}\left\langle\frac{1}{2}\left(1+*_{\tilde{\omega}}\right)\left(\chi_{j}+d\left(\frac{\partial b}{\partial s_{j}}\left(\underline{0}, t_{0}\right)\right)\right), \tilde{\chi}_{i}\right\rangle_{\tilde{\omega}} \frac{\tilde{\omega}^{2}}{2} \\
& =\int_{M} \chi_{j} \wedge \tilde{\chi}_{i},
\end{aligned}
$$

which is invertible. Applying the implicit function theorem again we find $\underline{s}=\underline{s}(t)$ solving $\Psi_{2}(\underline{s}(t), t)=0$ and hence

$$
\Phi(b(\underline{s}(t), t), \underline{s}(t), t)=0,
$$

for $t$ close to $t_{0}$. This completes the proof of openness.

We now turn to the question of closedness. As discussed in section 6 , we may assume that that $N(J)$ is small in the $L^{p}$ sense for any fixed $p$. Assume that we have a solution of (7.1) with $\int_{M} \omega_{t}^{\prime} \wedge \omega>0$ and $\left[\omega_{t}^{\prime}\right] \in H_{\omega}^{+}$on some maximal interval $\left[0, t_{0}\right)$. Write $\omega^{\prime}=\omega_{t}^{\prime}$ and define $s_{i}$ by $\left[\omega^{\prime}\right]=[\omega]+\sum_{i=0}^{r} s_{i}\left[\chi_{i}\right]$ for $\chi_{0}=\omega$ and $\chi_{1}, \ldots, \chi_{r}$ as above. Notice that by squaring both sides of this equation we see that the $s_{i}$ are bounded. Define $\phi_{0}$ and $\phi_{1}$ by

$$
\begin{aligned}
\frac{1}{4} \Delta \phi_{0} & =\frac{\omega \wedge \omega^{\prime}}{\omega^{2}}-\int_{M} \omega^{\prime} \wedge \omega \\
\frac{1}{4} \Delta^{\prime} \phi_{1} & =\int_{M} \omega^{\prime} \wedge \omega-\frac{\omega \wedge \omega^{\prime}}{\omega^{2}},
\end{aligned}
$$

where we recall that $\int_{M} \omega^{2}=\int_{M} \omega^{\prime 2}=1$. Let us first assume that $\phi_{1}$ is uniformly bounded. Then since $\int_{M} \omega^{\prime} \wedge \omega=1+s_{0}>0$ is uniformly bounded from above, Theorem 3.1 still holds with essentially the same 
proof. Notice that the bound on $\operatorname{tr}_{g} g^{\prime}$ implies a uniform positive lower bound for $\int_{M} \omega^{\prime} \wedge \omega$. No changes are necessary for section 4 . For the higher order estimates, we argue as follows. Define $a_{0}$ by $d^{*} a_{0}=0$ and

$$
\omega^{\prime}=\omega+\sum_{i=0}^{r} s_{i} \chi_{i}-\frac{1}{2} d\left(J d \phi_{0}\right)+d a_{0} .
$$

Then $a_{0}$ satisfies the equations

$$
\begin{aligned}
d a_{0} \wedge \omega & =-\sum_{i=1}^{r} s_{i} \chi_{i} \wedge \omega \\
\mathcal{P} d a_{0} & =-\sum_{i=1}^{r} s_{i} \mathcal{P} \chi_{i}+\frac{1}{4}\left(\partial_{i} J_{j}{ }^{k}-\partial_{j} J_{i}{ }^{k}\right)\left(\partial_{k} \phi_{0}\right) d x^{i} \wedge d x^{j},
\end{aligned}
$$

and the arguments of section 5 follow in just the same way as before.

We will now show that $\phi_{1}$ can be bounded if $N(J)$ is small in the $L^{p}$ norm for $p>2$. Define $a_{1}$ by

$$
\omega^{\prime}=\omega+\sum_{i=0}^{r} s_{i} \chi_{i}-\frac{1}{2} d\left(J d \phi_{1}\right)+d a_{1},
$$

and $d_{1}^{*} a_{1}=0$ where we are using the subscript 1 to denote the metric $\omega^{\prime}$. For ease of notation, set

$$
\zeta=\frac{1}{4}\left(\partial_{i} J_{j}^{k}-\partial_{j} J_{i}^{k}\right)\left(\partial_{k} \phi_{1}\right) d x^{i} \wedge d x^{j}
$$

The 1-form $a_{1}$ satisfies

$$
\begin{aligned}
d a_{1} \wedge \omega^{\prime} & =-s_{0} \omega^{\prime 2}-\sum s_{i} \chi_{i} \wedge \omega^{\prime} \\
\mathcal{P} d a_{1} & =\zeta-\mathcal{P}\left(\sum s_{i} \chi_{i}\right),
\end{aligned}
$$

where, here and from now on, we are always summing $i$ from 0 to $r$. This equation can be rewritten as

$$
d_{1}^{+} a_{1}=\zeta-s_{0} \omega^{\prime}-\frac{1}{2}\left(1+*_{1}\right) \sum s_{i} \chi_{i} .
$$

Write $\Pi$ for the $L^{2}\left(\omega^{\prime}\right)$ projection onto the space $\mathcal{H}_{\omega^{\prime}}^{+}$of self-dual harmonic forms with respect to $\omega^{\prime}$. Then we see that

$$
\begin{aligned}
(1-\Pi) \zeta & =d_{1}^{+} a_{1}+(1-\Pi) \frac{1}{2}\left(1+*_{1}\right) \sum s_{i} \chi_{i} \\
\Pi \zeta & =s_{0} \omega^{\prime}+\Pi \sum s_{i} \chi_{i}=\Pi \sum \tilde{s}_{i} \chi_{i},
\end{aligned}
$$

for $\tilde{s}_{0}=s_{0}\left(2+s_{0}\right)$ and $\tilde{s}_{i}=s_{i}\left(1+s_{0}\right)$ for $i \geq 1$. Now Lemma 6.1 holds as before if we replace (6.2) by the inequality

$$
\left(\int_{M}\left|\frac{d a_{1} \wedge \omega}{\omega^{2}}\right|^{p} \omega^{2}\right)^{\frac{1}{p}}+\left(\int_{M}\left|\frac{\sum s_{i} \chi_{i} \wedge \omega}{\omega^{2}}\right|^{p} \omega^{2}\right)^{\frac{1}{p}} \leq B
$$


where we are making use of the fact that $\left(d a_{1}+\sum s_{i} \chi_{i}\right) \wedge \omega^{\prime}=-s_{0} \omega^{\prime 2}$ is bounded. We can now replace the inequality (6.4) in the Claim by

$$
\left(\int_{M}\left|\frac{d a_{1} \wedge \omega}{\omega^{2}}\right|^{p} \omega^{2}\right)^{\frac{1}{p}}+\left(\int_{M}\left|\frac{\sum s_{i} \chi_{i} \wedge \omega}{\omega^{2}}\right|^{p} \omega^{2}\right)^{\frac{1}{p}}<1 .
$$

Indeed, arguing for a contradiction as in the proof of the claim, we suppose that we have equality in (7.6). Then $\omega^{\prime}$ and $\omega$ are uniformly equivalent and we can essentially ignore the fact that they define different norms. Writing $C$ for a uniform constant which may change from inequality to inequality we have $\|\zeta\|_{L^{p}} \leq C\|N(J)\|_{L^{p}}$ from which it follows that $\|\Pi \zeta\|_{L^{2}} \leq C\|N(J)\|_{L^{p}}$. Then we see from (7.5) that $\left|\tilde{s}_{i}\right| \leq C\|N(J)\|_{L^{p}}$. Hence $\left|s_{i}\right| \leq C\|N(J)\|_{L^{p}}$ and

$$
\left\|\sum s_{i} \chi_{i}\right\|_{L^{p}} \leq C\|N(J)\|_{L^{p}}
$$

But we also have $\|\Pi \zeta\|_{L^{p}} \leq C\|N(J)\|_{L^{p}}$ and hence $\|(1-\Pi) \zeta\|_{L^{p}} \leq$ $C\|N(J)\|_{L^{p}}$. Then from (7.4) and the elliptic $L^{p}$ estimates we have

$$
\left\|d a_{1}\right\|_{L^{p}} \leq C\|N(J)\|_{L^{p}} .
$$

Choosing $\epsilon$ sufficiently small, we obtain the contradiction from (7.7) and (7.8).

q.e.d.

\section{References}

[Au] T. Aubin, Équations du type Monge-Ampère sur les variétés kählériennes compactes, Bull. Sci. Math. (2) 102(1) (1978) 63-95, MR 0494932, Zbl 0374.53022.

[BaKo1] S. Bando \& R. Kobayashi, Ricci-flat Kähler metrics on affine algebraic manifolds, in 'Geometry and Analysis on Manifolds', Lecture Notes in Mathematics, 1339, 20-31, Springer-Verlag, 1988, MR 0961470, Zbl 0659.53048 .

[BaKo2] _ Ricci-flat Kähler metrics on affine algebraic manifolds, II, Mathematische Annalen 287 (1990) 175-180, MR 1048287, Zbl 0701.53083.

[Ca] E. Calabi, The space of Kähler metrics, in 'Proceedings of the International Congress of Mathematicians', Amsterdam, 1954, Vol. 2, 206-207, NorthHolland, Amsterdam, 1956, MR 0070535.

[De] P. Delanoë, Sur l'analogue presque-complexe de l'équation de Calabi-Yau, Osaka J. Math. 33(4) (1996) 829-846, MR 1435456, Zbl 0878.53030.

[Do] S.K. Donaldson, Two-forms on four-manifolds and elliptic equations, in preparation.

[DoKr] S.K. Donaldson \& P.B. Kronheimer, The geometry of four-manifolds, Clarendon Press, Oxford, 1990, MR 1079726, Zbl 0820.57002.

[Ev] L.C. Evans, Classical solutions of fully nonlinear, convex, second order elliptic equations, Comm. Pure Appl. Math. 25 (1982) 333-363, MR 0649348, Zbl 0469.35022. 
[FeGoGr] M. Fernández, M. Gotay, \& A. Gray, Compact parallelizable four-dimensional symplectic and complex manifolds, Proc. Amer. Math. Soc. 103(4) (1988) 1209-1212, MR 0955011, Zbl 0656.53034.

[GiTr] D. Gilbarg \& N.S. Trudinger, Elliptic partial differential equations of second order, Grundlehren der Mathematischen Wissenschaften, 224, Springer-Verlag, Berlin, 1983, MR 0737190, Zbl 0562.35001.

[Gr] M. Gromov, Pseudo holomorphic curves in symplectic manifolds, Invent. Math. 82 (1985) 307-347, MR 0809718, Zbl 0592.53025.

[Ko] S. Kolodziej, The complex Monge-Ampère equation, Acta Math. 180(1) (1998) 69-117, MR 1618325, Zbl 0913.35043.

[Kr] N.V. Krylov, Boundedly nonhomogeneous elliptic and parabolic equations, Izvestia Akad. Nauk. SSSR 46 (1982) 487-523; English translation in Math. USSR Izv. 20(3) (1983) 459-492, MR 0661144, Zbl 0529.35026.

[Jo] D. Joyce, Compact manifolds with special holonomy, Oxford Mathematical Monographs, Oxford University Press, Oxford, 2000, MR 1787733, Zbl 1027.53052.

[MoWa] I. Motzkin \& W. Wasow, On the approximation of linear elliptic differential equations by difference equations with positive coefficients, J. Math. Phys. 31 (1952) 253-259, MR 0052895, Zbl 0050.12501.

[Si] Y.-T. Siu, Lectures on Hermitian-Einstein metrics for stable bundles and Kähler-Einstein metrics, DMV Seminar, 8, Birkhauser Verlag, Basel, 1987, MR 0904673, Zbl 0631.53004.

[Th] W. Thurston, Some simple examples of symplectic manifolds, Proc. Amer. Math. Soc. 55 (1976) 467-468, MR 0402764, Zbl 0324.53031.

[TiYa1] G. Tian \& S.-T. Yau, Complete Kähler manifolds with zero Ricci curvature I, J. Amer. Math. Soc. 3(3) (1990) 579-609, MR 1040196, Zbl 0719.53041.

[TiYa2] Complete Kähler manifolds with zero Ricci curvature II, Invent. Math. 106(1) (1991) 27-60, MR 1123371, Zbl 0766.53053.

[Tr1] N.S. Trudinger, Local estimates for subsolutions and supersolutions of general second order elliptic quasilinear equations, Invent. Math. 61 (1980) 67-79, MR 0587334, Zbl 0453.35028.

[Tr2] Fully nonlinear, uniformly elliptic equations under natural structure conditions, Trans. Amer. Math. Soc. 278(2) (1983) 751-769, MR 0701522, Zbl 0518.35036.

[Ya] S.-T. Yau, On the Ricci curvature of a compact Kähler manifold and the complex Monge-Ampère equation, I, Comm. Pure Appl. Math. 31(3) (1978) 339-411, MR 0480350, Zbl 0369.53059.

HARVARD UNIVERSITY

Department of Mathematics

Cambridge, MA 02138

E-mail address: weinkove@math.harvard.edu 